\title{
The interaction between two oppositely travelling, horizontally offset, antisymmetric quasi-geostrophic hetons
}

\author{
By JEAN N. REINAUD ${ }^{1}$ AND XAVIER CARTON ${ }^{2}$ \\ ${ }^{1}$ Mathematical Institute, University of St Andrews, North Haugh, St Andrews, KY16 9SS, UK \\ ${ }^{2}$ Laboratoire de Physique des Océans, UFR Sciences, UBO/UEB, 6, Avenue le Gorgeu, 29200 \\ Brest, France
}

(Received 26 February 2016)

We investigate numerically the nonlinear interactions between hetons. Hetons are baroclinic structures consisting of two vortices of opposite sign lying at different depths. Hetons are long-lived. They most often translate (they can sometimes rotate) and therefore they can noticeably contribute to the transport of scalar properties in the oceans. Heton interactions can interrupt this translation and thus this transport, by inducing a reconfiguration of interacting hetons into more complex baroclinic multipoles. More specifically, we study here the general case of two hetons, which collide with an offset between their translation axes. For this purpose, we use the point vortex theory, the ellipsoidal vortex model and direct simulations in the three-dimensional quasi-geostrophic, contour surgery model. More specifically, this paper shows that there are in general three regimes for the interaction. For small horizontal offsets between the hetons, their vortices recombine as same-depth dipoles which escape at an angle. The angle depends in particular on the horizontal offset. It is a right angle for no offset, and the angle is shallower for small but finite offsets. The second limiting regime is for large horizontal offsets where the two hetons remain the same hetonic structures but are deflected by the weaker mutual interaction. Finally the intermediate regime is for moderate offsets. This is the regime where the formation of a meta-stable quadrupole is possible. The formation of this quadrupole greatly restrains transport. Indeed, it constrains the vortices to reside in a closed area. It is shown that the formation of such structures is enhanced by the quasi periodic deformation of the vortices. Indeed, these structures are nearly unobtainable for singular vortices (point vortices) but may be obtained using deformable, finite-core vortices.

\section{Introduction}

Mesoscale eddies play a key role in the transport of momentum, heat and nutrients in the oceans. It is currently estimated that about half of the overall transport in the oceans may be attributed to mesoscale vortices, see Zhang, Wang and Qiu (2014). The ubiquity of vortices in the oceans is well documented in the literature, see for example Chelton et al. (2007). A typical survey of the vortex population in the surface layer of the North Atlantic alone proposed by Ebbesmeyer et al. (1986) estimates that between 1,000 to 10,000 structures may be found at any time. Although this estimate is an extrapolation from sample measurement is a small region of the Atlantic, a plethora of satellite measurements and imagery confirms the ubiquity of the coherent structures (Chelton, Schlax and Samelson, 2011). An isolated vortex does not induce an overall advection on itself, but rather spins. Vortices, however, advect as a result of their interactions. Vortices may 
interact with coastal boundaries, jets and currents as well as with bottom topography. More importantly vortices interact with one another. Vortex interaction has been extensively studied in the literature, in many different contexts, over very large parameter spaces, see Carton (2001) for a review.

Within the context of ocean dynamics, flows are strongly affected by the stable stratification of the waters, and by the Earth's rotation. The simplest dynamical model to study fluid flows in this situation is the quasi-geostrophic model. In this paper, we will focus on the three-dimensional, incompressible quasi-geostrophic model. In this context, vortices are best defined as compact volumes of anomalous potential vorticity. Previous studies include vortex merger, see von Hardenburg et al (2000), Dritschel (2002) or Reinaud \& Dritschel (2002) and sub-sequent studies, as well as destructive interactions for dipoles, see Reinaud and Dritschel (2009). This has been done solving the full quasi-geostrophic equations or using simplified models, such as the Ellipsoidal Model, see Reinaud and Dritschel (2005).

Hetons are another kind of vortex structures which are observed in the oceans. Hetons consist of two opposite signed vortices lying at different depths. Several formation mechanisms are possible such as the presence of local surface cooling (ice) and brine (see Chao and Shaw, 1999), or the baroclinic instability of coastal jets (Cherubin et al , 1997) and the destabilisation deep jets (see Flierl, Carton and Messager, 1999), see also Sokolovskiy and Verron (2014) for a discussion. Such structures were originally described for point vortices or charges by Gryanik $(1983 \mathrm{a}, \mathrm{b})$ in a two-layer model and continuous stratification respectively. Independently, Kizner (1984) introduced Rossby solitons which are essentially hetons as well. The term hetons was however introduced in the seminal paper by Hogg and Stommel (1985). Again, the authors first focused on singular vortices. It should be noted that similar problems were also investigated by Young (1985). Finite-core hetons and their stability has been addressed in a series of papers, both in layered models and in continuous stratification. Kozlov, Marakov and Sokolovskiy (1986), Helfrich and Send (1988), and Flierl (1988) studied the stability of two-layer hetons whose vortices are aligned in the vertical direction, while for example Sokolovskiy (1997) studied hetons in a three-layer model. An extensive overview and detailed bibliography for the problem in a two-layer model may be found in Sokolovskiy and Verron (2014). The existence of singular (explosive) modes for the two-layer problem was addressed by Carton et al. (2010) while the parametric instability for the same problem is addressed in Carton at al. (2010b). Kizner, Berson and Khvoles $(2002,2003)$ also studied smooth hetons (referred to as baroclinic modons) on the $\beta$-plane. Their solutions can be adapted to the $f$-plane. For continuous stratification, the problem is discussed in Reinaud and Carton (2009) in the case where the vortices are aligned in the vertical direction and where the vortices may be separated by a vertical gap. Misaligned vortices, referred to as 'tilted' hetons in the literature, are addressed in Reinaud (2015). The overall upshot of all these studies is that hetons may be sensitive to baroclinic instability. The instability depends on the aspect ratio of the vortices constituting the hetons. Wider vortices are sensitive to higher azimuthal wavenumber modes of perturbation. The instability may be suppressed if the vortices are compact and/or well separated either or both in the vertical direction and in the horizontal direction. This means that compact-core, well separated hetons are robust (stable) and may be efficient candidates to transport flow properties over long time, and subsequently long distances.

Because hetons move in the flow (provided their vortices are horizontally offset), they may enter the vicinity of other vortices and in particular other hetons. Indeed the interaction between hetons has been observed in the oceans, for example in L'Hegaret et al. (2014). The motion and the interactions between hetons has been studied in the litera- 
ture, mostly within the context of two-layer models. The interaction between the hetons may redistribute potential vorticity by, for example, breaking and/or merging vortices. Studies in the two-layer context include Valcke and Verron (1993), Sokolovskiy and Verron (2000), Gryanik, Sokolovskiy and Verron (2006) and Kizner (2006) and references therein to give a non-exhaustive list.

The interaction between two continuously stratified hetons, with possibly a vertical gap between the vortices is addressed in Reinaud and Carton (2015a) for the case where the two colliding hetons are initially translating along the same axis, in opposite directions. It is shown that the interaction between the hetons may compete with the intrinsic stability of the hetons to baroclinic modes. These interactions may produce further multipolar structures and small scale debris and filaments. Moreover, at leading order, even for stable hetons, the interaction may result in significant changes in the hetons trajectories. The hetons may recombine and escape at an angle nearly perpendicular to the initial trajectories. Alternatively, the hetons may reverse their trajectory and further separate at long time. These interactions depends on the region of the parameter space identified in Reinaud and Carton (2015a), and in particular on the set-up of the hetons. Indeed for two hetons to translate towards one another, two different set-up are possible. In the one case, the vortices lying at the same depth (one vortex from each incoming heton) have the same polarity. This case is referred to as the symmetric case. The second case corresponds to the case where the vortices lying at the same depth have different polarities, referred to as the antisymmetric case. When the two hetons are additionally offset in the horizontal but still initially translate along parallel axes, a symmetric pair of hetons may recombine to form a tripolar structure. Such structures are investigated in Reinaud and Carton (2015b).

The present study addresses the interaction between two antisymmetric hetons when the hetons are initially horizontally offset from one another. This gives rise to potential different behaviours from the aligned case studied in Reinaud and Carton (2015a), as the hetons no longer collide 'head-on'. In particular the behaviour depends on how much the hetons are offset (as a function of the other parameters of the flow). We show that the behaviour of the interacting antisymmetric hetons may fall into one of the three generic classes. For small horizontal offsets, the interaction is qualitatively similar to the one of aligned hetons. In such scenarios, the vortices of the hetons recombine as same-depth dipoles and escape at an angle. For large horizontal offsets the hetons are too distant from one another to strongly interact together, and the overall result of the weaker interaction results in a deflection of the initial trajectories. The intermediate regime for moderate horizontal offsets is dynamically richer. There is a meta-stable solution (originated by the existence of a true equilibrium) where the vortices form a quadrupole which rotate about the centre of the system. These structures are referred to as Z-vortices, due to the shape the vortices exhibit, Sokolovskiy and Carton (2010). This, as a consequence, alters drastically the transport as the vortices remain confined in a localised area. Moreover, in our case, the overall strength of the quadrupoles is zero as there is as much negative potential vorticity as positive one. This means that the velocity field induced outside the quadrupole decays very rapidly away from it. As a consequence, the quadrupole rotates within an overall quiet environment.

The complete problem depends on a large number of parameters covering a huge parameter space. For finite-core vortices, even when imposing equal volume and general shape (aspect-ratio), and equal potential vorticity (in absolute value) for the four vortices, the problem still depends on four parameters: the aspect ratio of the vortices, the vertical offset between the vortices, and two horizontal offsets, one between the vortices within each hetons, and one between the two hetons. A comprehensive study solving the full 
quasi-geostrophic equations is out of reach. It is however possible to draw the main, generic, dynamical features of the interaction. To do this we propose a methodology for studying the problem using hierarchy of models. We shall start by general considerations based on arguments on the velocities of singularities at an instant frozen in time, as well as the existence of a configuration in equilibrium. Then, the explicit time-integration of the singularities motion reveals the possible evolutions for the pair of hetons colliding from a distance. It is shown however that the practical formation of a Z-vortex necessitates the ability for the vortices to deform, and adapt. At leading order, the influence of the shape of the vortices may be studied using an asymptotic model: the Ellipsoidal Model, see Dritschel, Reinaud and McKiver (2004). Finally the full dynamics of the interaction has been studied on a large number of cases to illustrate the outcome.

The paper is organised as follows. $\S 2$ describes the equations used and the general set-up of the problem. $\S 3$ discusses the possible trajectories for the hetons using singularities and indicate the existence of equilibrium solutions in this context. $\S 4$ discussed the intermediate regime where Z-vortices can be obtained. This section includes discussions on the finite-core effects using the Ellipsoidal Model. It also addresses the full dynamics, and in particular the formation of small scales and debris. $\$ 5$ revisits the non destructive interaction using the full dynamics, where the hetons recombine or are deflected. Conclusions are drawn in $\S 6$. The paper is completed by 3 appendices addressing technical details, and a summary of the outcome of large number of nonlinear simulations from the large parameter space of the problem.

\section{Equations of motion and problem set-up}

We investigate the evolution of vortices under the quasi-geostrophic approximation. This approximation derives from a Rossby number expansion of the primitive equations and is strictly valid for $F r^{2} \ll R o \ll 1$. Here, the Froude number $F r=U /(N H)$ is the ratio of a scale of horizontal vorticity $U / H$ where $U$ is a typical horizontal velocity scale and $H$ a vertical length to the buoyancy frequency $N$ in the stably stratified fluid. The Rossby number $R o=U /(f L)$ is the ratio of $U / L$ which scales as the vertical component of the relative vorticity ( $L$ is a typical horizontal scale), to $f$ is the Coriolis frequency. We assume in the study that both $N$ and $f$ are constant for the sake of simplicity. Stretching the physical vertical coordinate $z^{*}$ by the constant ratio $N / f$, the governing equations read

$$
\begin{gathered}
\Delta \psi=q . \\
u=-\frac{\partial \psi}{\partial y} \quad \text { and } \quad v=\frac{\partial \psi}{\partial x}, \\
\frac{D q}{D t}=0,
\end{gathered}
$$

where $D / D t=\partial / \partial t+\mathbf{u} \cdot \boldsymbol{\nabla}=\partial / \partial t+J(\psi, \cdot)$ is the material derivative. $q$ is the potential vorticity anomaly, hereinafter referred to as $\mathrm{PV}$, and $\psi$ is the streamfunction. In the first equation, the operator $\Delta$ is the three-dimensional Laplacian where the vertical coordinate $z=z^{*} N / f$, and $\Delta=\partial^{2} / \partial x^{2}+\partial^{2} / \partial y^{2}+\partial^{2} / \partial z^{2}$. The advection velocity field $\mathbf{u}=$ $(u, v)$ is the geostrophic velocity field, and although the vertical velocity $w$ is not zero in quasi-geostrophy, it is small enough not to contribute to the advection of the potential vorticity $q$. Details on quasi-geostrophic equations originated by Charney (1947) and their derivation may be found in Vallis (2006) for example.

The general geometry of the configuration is described in figure 1. Each heton consists of a pair of vortices of equal size and equal and opposite PV lying at different depths. 


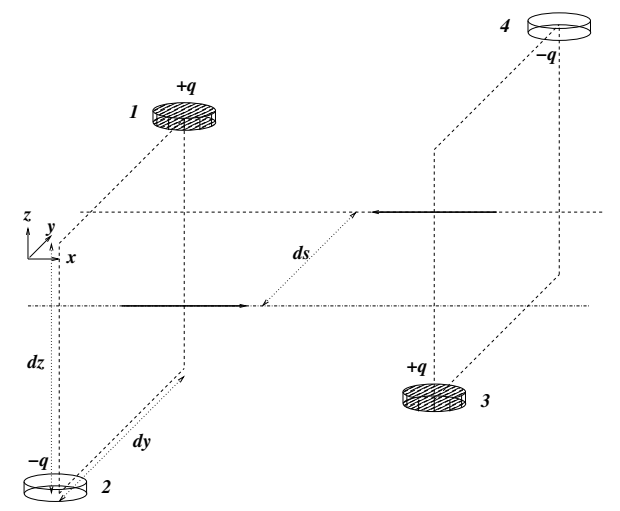

FiguRE 1. Geometry of the antisymmetric horizontally offset hetons. The vortices are labelled as 1 (top left) 2, (bottom left), 3 (bottom right) and 4 (top right). vortices 1 and 2 constitute the first heton while vortices 3 and 4 form the second one. vortices 1 and 3 have positive sign $+q$ while vortices 2 and 4 are negative $(-q) . d z$ and $d y$ indicate the vertical and horizontal offsets within each heton. $d s$ is the horizontal offset between the two hetons. The bold arrows indicate the initial direction of translation velocity.

For the sake of simplicity the two hetons are similar. The vortices within each heton are initially aligned along the $x$-direction and offset in the horizontal in the $y$-direction such that each heton (individually) translates along the $x$-axis. The horizontal offset, in combination with the polarity of the vortices is chosen such that the hetons initially move toward one another. This set-up is the same as the one used in Reinaud and Carton (2015a) except the two hetons initially do not travel along the same axis but along parallel axes (the axes are horizontally offset). In this paper, we focus on the interaction between 'antisymmetric hetons', following the nomenclature in Reinaud and Carton (2015a). This means that the two vortices lying at the same depth (each belonging to one of the hetons) have opposite polarity. The horizontal offset puts the two positive vortices (which lie at different depth) closer horizontally while placing the two negative ones further away from one another. Because the volume and the absolute value of $\mathrm{PV}$ is set to be the same for each of the four vortices, the first parameters which can be varied are $d y$, the horizontal offset between the vortices within each heton, $d z$ their vertical offset. Then, $d s$ denotes the horizontal offset between the two hetons. Finally we can choose the aspect ratio of the vortices $\varrho=r / h$, where $r$ is the mean horizontal radius of the vortices, and $h$ is the total height of the vortices. For the full dynamics, the vortices are initially set to be cylinders.

\section{Point vortices and possible trajectories}

\subsection{Interactions between two distant incoming hetons}

We first discuss the possible trajectories for the interacting antisymmetric hetons. We illustrate their behaviour modelling the vortices by singularities. This approach allows to investigate very rapidly large parameter spaces, as the computational cost is minimal. Doing so, we however lose the shape of the vortices and the aspect ratio $\varrho$ of the vortices is no longer a parameter. The three parameters remaining are the three offsets $d y, d z$ and $d s$. Without loss of generality, only two non-dimensional length ratios are independent, for example $d z / d y$ and $d s / d y$.

In the case $d s=0$, when the two hetons are facing each other, the location of the 4 vortices may be determined from the location of one of the vortices only, using symmetry 
for the other 3, as discussed in Reinaud and Carton (2015). This allows to determine the possible trajectories of the vortices without solving the time dependent dynamical equations of motion for the singularities, but using instead the spatial structure of the Hamiltonian function alone (see the discussion in Reinaud and Carton, 2015). This is no longer possible in the present case, as we break the symmetry which allows to determine the position of the 3 other point vortices at all times from the knowledge of the position of one alone. The time-dependent dynamical equations have to be solved for each case (i.e. for each $d z / d y$ and each $d s / d y$ ). This also allows for more, non-trivial trajectories. In particular there are small regions of the parameter space where the advection of the singularities is highly sensitive to the initial conditions.

Despite these complex behaviours, we may distinguish 3 general regimes, depending on the ratio $d s / d y$. As seen in Reinaud \& Carton (2015a), in the case $d s=0$, the only generic behaviour observed for singularities is the recombination of the vortices of the hetons into dipoles formed by the opposite-signed vortices lying at the same depth. When $d s=0$, the dipoles escape nearly at a right angle from the original trajectories of the incoming hetons. This behaviour does not qualitatively depend on the aspect ratio $d z / d y$ characterising the incoming hetons. For $d s=0$, the incoming hetons always get close enough to one another such that the same-depth vortices, which initially belong to different hetons, recombine as dipoles. It is expected that for small values of the ratio $d s / d y$, i.e. small horizontal offsets between the two hetons, the behaviour will be similar, and the vortices will recombine as dipoles escaping at an angle depending on the initial conditions. In other words, the first regime we may expect is the recombination of hetons into dipoles for small $d s / d y$.

For large relative horizontal offsets $d s / d y$, the two hetons are well separated in the horizontal. Keeping in mind that the overall strength associated with each heton is zero (the positive vortex compensates the negative one), the influence of a heton on the other one decreases rapidly with their separation distance. As a consequence, the vortices of one heton will not strongly interact with the vortices of the other heton. The weak influence of one heton onto the other one will merely result in the deflection of the trajectories. We may say that the second regime is the deviation or deflection of the hetons for large $d s / d y$.

The complex regime is the intermediate one, i.e. for $d s / d y \simeq \mathcal{O}(1)$. In this case, the respective influence of all singularities is likely to compete with each other. As mentioned before, the actual trajectory of the singularities can only be obtained by the explicit time integration of the evolution equations. However, a rapid point vortex calculation of the velocities at a frozen instant of time allows to better understand the competition between the various effects. For this purpose we consider the velocity $v_{1}$ in the $y$-direction of the inner, upper vortices labelled 1 in figure 1 . This corresponds to one of the vortices being put closer in the horizontal direction to its counterpart in the other heton. Following the geometry of the configuration, for $v_{1}<0$ vortex 1 goes towards the vortex of opposite sign lying at the same depth of the second heton (labelled vortex 4 in figure 1). This indicates the likeliness of the formation of a same depth dipole. For $v_{1}>0$, vortex 1 goes away from vortex 4 indicating the likeliness of the deflection of the heton away from the second heton. The details of the calculation of $v_{1}$ for given $d y, d z$, and $d s$ is proposed in the Appendix I, and the result reads

$$
v_{1}>0, \text { iff } \frac{d s}{d y}>\lambda_{c} \equiv \frac{1+(d z / d y)^{2}}{2},
$$

and $v_{1} \leqslant 0$ otherwise. In fact this corresponds to the competition of the influence of 

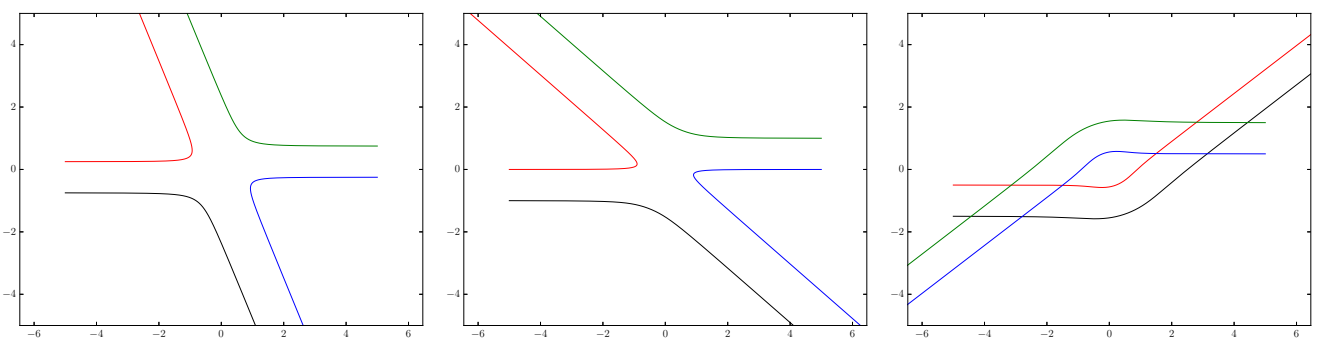

FiguRE 2. Top view of the trajectories of two incoming point vortex hetons for $d z=1, d y=1$ and $d s=0.5$ (left), $d s=1$ (centre) and $d s=2$ (right). At $t=0$, the singularities are located at $x= \pm 5$.

vortices 3 and 4 over vortex 1 . The former case corresponds to the case where vortex 4 is closer to vortex 1 than vortex 3 is. Since vortices 3 and 4 have equal and opposite strength, in that case vortex 4 dominates the interaction. The latter case corresponds to the case where vortex 3 is closer to vortex 1 . It should be noted that this calculation does not fully represent what happens during the actual time-dependent nonlinear evolution as the calculation assumes that the singularities are located following a pattern similar to the initial conditions. This arrangement is no longer exactly valid at $t>0$ as the relative position of the vortices may change. In particular, the vortices of each heton are no longer aligned along the $y$-direction because their velocities $u$ is not equal. However, the model illustrates the dynamics at the leading order. For $2 d s / d y \ll 1+(d z / d y)^{2}$ one expects the hetons to recombine as dipoles while for $2 d s / d y \gg 1+(d z / d y)^{2}$ the hetons are expected to remain hetons which deviate from their initial trajectory. Finally for $2 d s / d y \sim 1+(d z / d y)^{2}$, vortices 3 and 4 have an influence of same relative importance. In this case another kind of interaction may occur. The vortices may stop translation and start rotating in a quasi-periodic way, at least temporarily. This specific regime is detailed in the following sections. We now illustrate the trajectories over 3 examples in figure 2 . We take $d z=1$ and $d y=1$ such that $\lambda_{c}=1$. In each case, we start the stimulation with point vortices distant by $d x=10$ in the $x$-direction. The equation of motions of the singularities are marched in time with a fourth order Runge-Kutta algorithm with $d t=0.01$. For reference, the strength of the singularities are set to $\pm 4 \pi$. The same numerical set-up is used for all point vortex numerical time integrations. Figure 2 (left) shows the trajectories for $d s / d y=0.5<\lambda_{c}$ for which we see that vortex 1 (inner vortex for the vortex on the left) escape and recombines with vortex 4 as a dipole. In figure 2 (right) we have $d s / d y=2>\lambda_{c}$ and the initially $v_{1}<0$, and vortex 1 starts to deviate to negative $y^{\prime} s$ and rotates around vortex 3 . However, having passed vortex 3 , the vortex continues its rotation and $v_{1}$ becomes positive again (one can see from the formula in the Appendix I that the sign of the dominant term for $v_{1}$ due to vortex 3 depends on the sign of $\left.x_{1}-x_{3}\right)$. Eventually the distance between the hetons increases again and the baroclinic structures resume a nearly linear trajectory.

Finally we consider a case at the threshold $d s / d y=1=\lambda_{c}$. This case is presented in figure 2 (centre). The outcome of the interaction is still the recombination of the two hetons into a pair of same depth dipoles, as for $d s<\lambda_{c}$. This is due to the non trivial relative displacement of the singularities. Indeed, the vortices within each heton do not retain their initial alignment in the $y$-direction due to the different relative distances between the vortices. As a consequence the full trajectory cannot be simply guessed from a situation frozen in time. We will go back to the intermediate regime between recombination as dipoles and deflections of the hetons in a subsequent section. Before 
doing this, we address the question of the existence of an intermediate solution where the influences of the different vortices balance in a way that the couple of hetons rotate rather than escaping away, either as dipoles (small $d s$ ) or hetons (large $d s$ ) in the next section.

\subsection{Existence of steadily rotating interacting hetons}

Sokolovskiy and Carton (2010) obtained the condition on the location of the vortices of the hetons for the hetons to steadily rotate within the context of a two-layer model. This would correspond to the situation where the two-layer configuration consists of a steady state in a uniformly rotating reference frame. We reproduce a similar calculation but in the three-dimensional, continuously stratified model. As in Sokolovskiy and Carton (2010), we look for the equilibrium of collinear vortices, meaning that the 4 vortices are aligned along a line (say the $x$-axis). One can write the equations of motion for the 4 singularities in a rotating reference frame with angular velocity $\Omega$. Then, we derive a compatibility condition on $d s / d z$ and $d y / d z$ such that there exists $\Omega$ for which the singularity are steady (in the rotating frame). Details of the calculation are presented in Appendix II, and the compatibility condition reads

$$
\begin{array}{r}
\left(\left(\frac{d s}{d z}\right)^{2}-\left(\frac{d y}{d z}\right)^{2}\right)\left(\frac{1}{\left(1+\left(\frac{d y-d s}{d z}\right)^{2}\right)^{3 / 2}}+\frac{1}{\left(1+\left(\frac{d y+d s}{d z}\right)^{2}\right)^{3 / 2}}\right) \\
=2\left(\frac{1}{(d s / d z)}-\frac{(d y / d z)^{2}}{\left(1+(d y / d z)^{2}\right)^{3 / 2}}\right) .
\end{array}
$$

This implicit relation $d s / d z$ versus $d y / d z$ is plotted in figure 3 (left). An alternative representation of the same relation but using $a \equiv(d s-d y) /(2 d z)$ and $b \equiv(d y+d s) /(2 d z)$ as parameters is proposed in figure 3 (right). The latter are the parameters equivalent to those used in Sokolovskiy and Carton (2010) for the two-layer problem. This double representation allows to clearly see two complementary asymptotic behaviours. From figure 3 (left) representing $d s / d z$ versus $d y / d z$, we see that $d s / d z \sim d y / d z$ for large $d s / d z$ (and $d y / d z$ ) at equilibrium. This corresponds to $a=(d s-d y) /(2 d z)$ small, i.e. the nearly vertical branch in figure 3 (right). In this case the two inner vortices are nearly aligned in the vertical while the outer vortices lie on each side. The second asymptotic behaviour is better seen in figure 3 (right), where we see $b \sim a$ for large values of $a$ and $b$ at equilibrium. Since $b-a=d y / d z, a \sim b$ corresponds to the nearly vertical branch seen on the left hand side of the figure, for small $d y$. In this configuration, each heton consists of two vortices nearly aligned in the vertical.

In our case, for $d y=1$, and $d z=1$ the compatibility condition for the existence of the equilibrium of collinear vortices gives $d s=1.361933905$. The trajectories of the vortices for this case are again computed by explicit time integration of the equations of motion, and are presented in figure 4 . We indeed recover a uniform rotation for the vortices. It is important to note that this configuration cannot be obtained from the initial conditions used for the interacting hetons incoming from further away. A simple argument is that the point vortices would have to lie on the circular trajectories at $t=0$. This is not consistent with the time evolution of two distant hetons travelling along parallel axes. However, the existence of a steadily rotating solution indicates the possibility of quasiperiodic rotation for the hetons provided they are attracted towards this configuration. This may be the case in the intermediate regime with $d s / d y \sim\left(1+(d z / d y)^{2}\right) / 2$. In that 

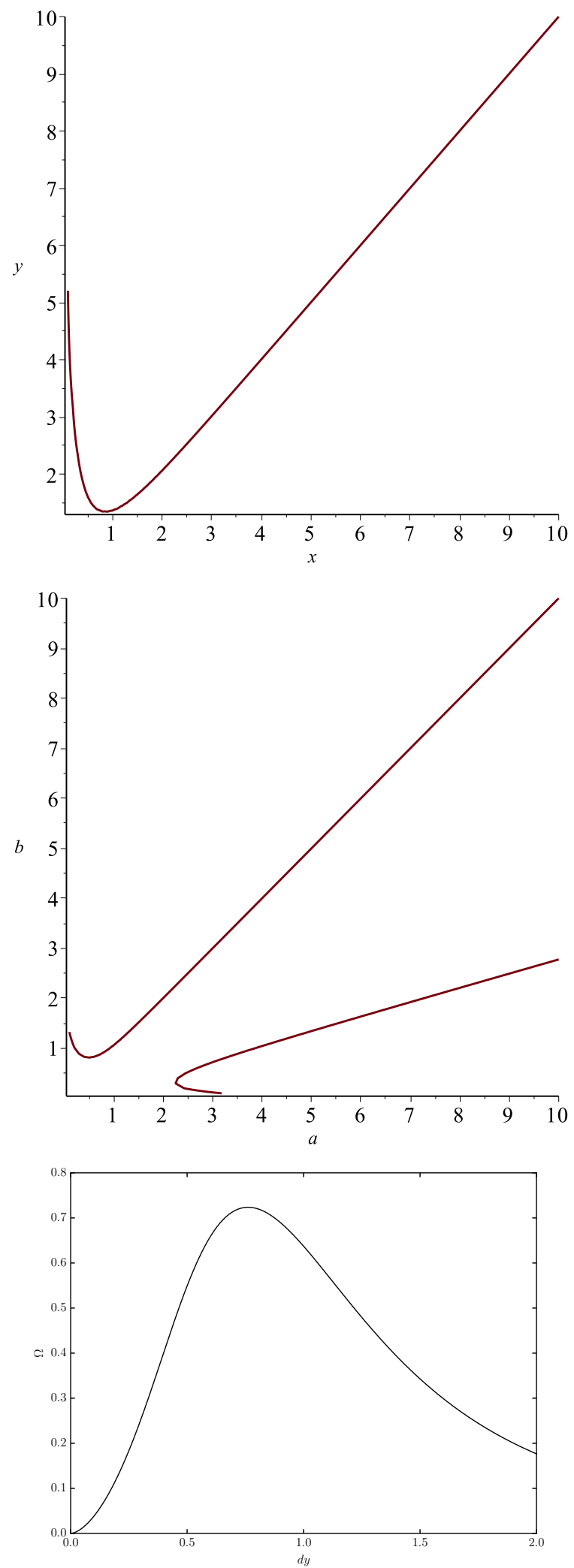

FIgURE 3. Compatibility condition for the existence of a collinear heton pair expressed as the relative horizontal offset between the two hetons $d s / d z$ as a function of the relative horizontal offset between the vortices within each heton $d y / d z$ (Top left). The figure on the top right gives the same condition but expressed in term of $a=(d s-d y) /(2 d z)$ vs $b=(d s+d y) /(2 d z)$ which are the parameters used Sokolovskiy and Carton (2010). The lower branch corresponds to $a>b$, i.e. $d s<0$. Bottom: the roation rate $\Omega$ of the equilivria vs $d y$. 


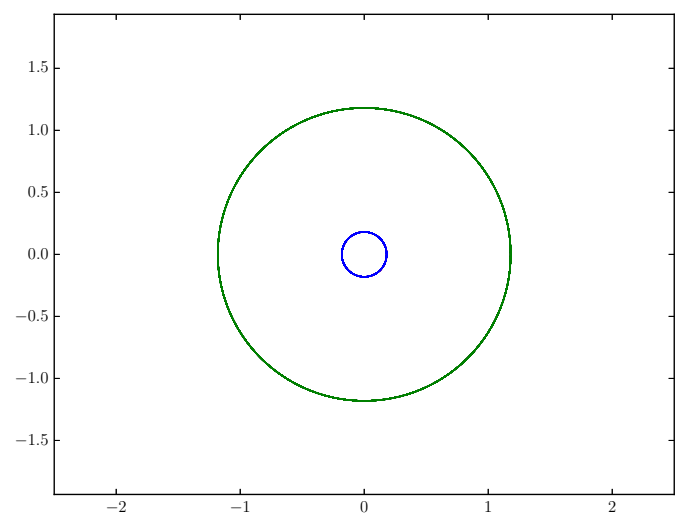

Figure 4. Top view of the trajectories for a collinear, steady rotating pair of hetons for $d z=1, d y=1$ and $d s=1.361933905$. At $t=0$, the singularities are located at $x= \pm 0$.

case, the hetons may reconfigure as a 4 vortices compound structure, already observed in Sokolovskiy and Carton (2010) and named 'Z-vortex' due to its apparent shape. This is the topic of the next section. In the case presented above, the quadupole of point vortices is in a linearly stable equilibrium. The results of the linear stability analysis for a wide range of values of $d y$ is presented in the Appendix II. It shows that there is in fact a region for small $d y<0.895$, where the equilibria is unstable.

\section{Formation of $Z$-vortices}

\subsection{Existence and effect of deformation}

In this section we investigate the possibility of forming a 'Z-vortex' from the interaction of two distant incoming hetons. We will first analyse in details an example which is generic of most cases of formation of a 'Z-vortex', before considering the process of formation over a larger parameter space. Again we start the investigation with the crudest, yet the fastest, model for the hetons using point vortices to represent the vortices. This allows to rapidly investigate the conditions under which the configuration starts to behave as a 'Z-vortex'. This is the case when the vortices start to exhibit circular trajectories rather than being merely deflected. As mentioned previously, although the exact circular trajectories exist (and are discussed in the previous section), they are inconsistent with the configuration of hetons incoming from a distance. However, we expect quasi-periodic motion to exist (at least temporarily) in this case. To investigate this possible behaviour, we set values for the vertical and horizontal offsets $d z$ and $d y$ within each heton and vary the parameter $d s$. We are searching for trajectories which exhibit the intiation of a global rotation. As mentioned before this regime should be an intermediate regime between the recombination of the vortices as dipoles (for small $d s$ ) and the deflection of the hetons (large $d s$ ). In other words one expects to obtain such configuration for $d s / d y=\mathcal{O}(1)$ (see both figure 3 for the actual 'Z-state', and the argument using the simple threshold $\left.d s / d y \sim\left(1+(d z / d y)^{2}\right) / 2\right)$. The two aforementioned criteria are not mathematically equivalent. Firstly $d s / d y=\left(1+(d z / d y)^{2}\right) / 2$ does not satisfy the compatibility criterion 3.2. The two criteria analyse two distinct phases in the process of formation of a Z-vortex. The criterion defined in (3.1) concerns the phase when the two hetons are moving closer together in the $x$-direction. On the other hand, the second criterion defined in (3.2) analyses the condition for uniform rotation then the vortices are collinear, i.e. when they have 'aligned' in the direction $y$ (constant $x$ ). Arguably, the first criterion allows to 

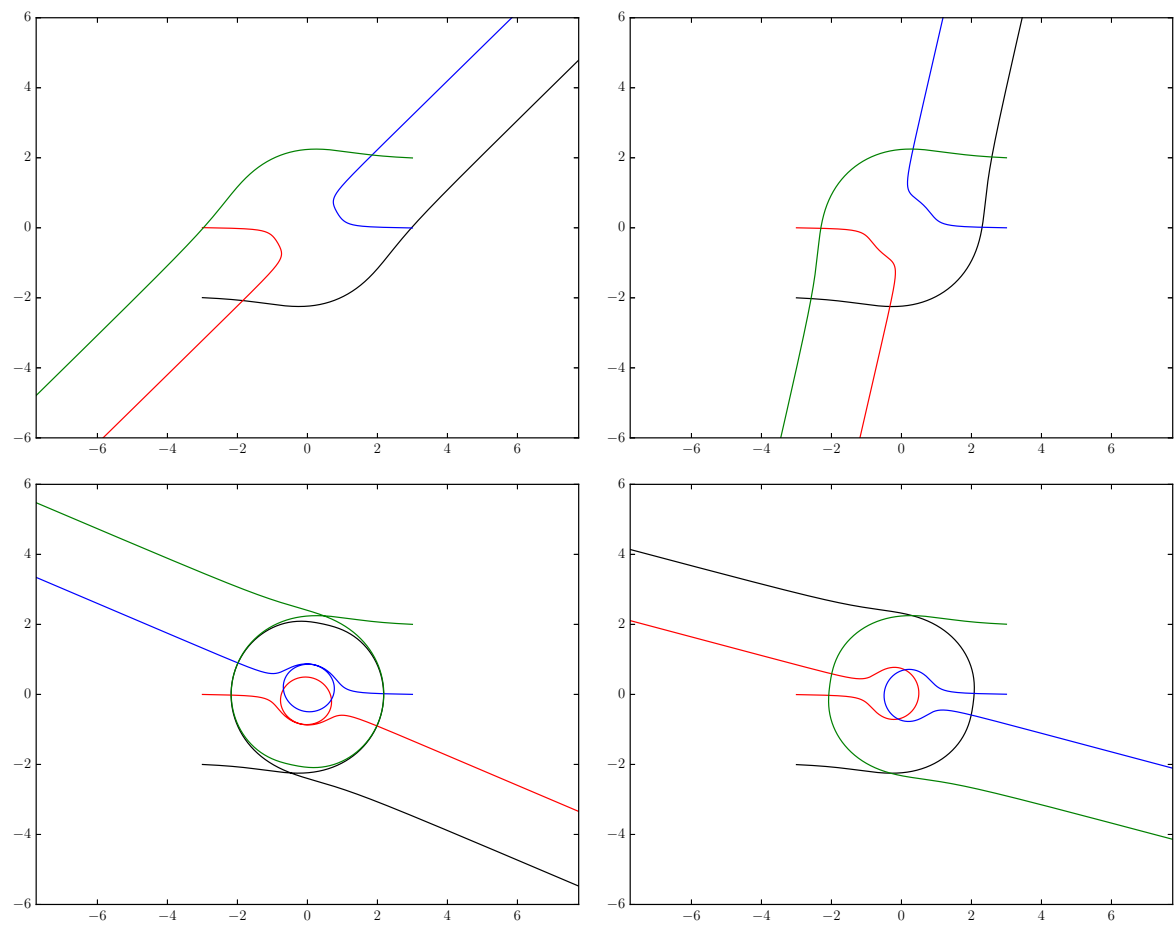

FiguRE 5. Top view of the trajectories of interacting, point vortices hetons with $\Delta z=1$, $\Delta y=2$ and $\Delta s=1.9901, \Delta s=2.0, \Delta s=2.0023$ and $\Delta s=2.01$. At $t=0, d x=5$.

distinguish between two asymptotic behaviours, $d s \ll d y$ and $d s \gg d y$, but is inconclusive for $d s \sim d y$. The second criterion provides some information is this situation.

We illustrate the trajectories of point vortex hetons in the case $d y / d z=2$ and some values of $d s / d z$. In each case, the hetons lie at a distance in the $x$-direction of $d x=5$ at $t=0$. Other examples have been investigated and may be found in Appendix III. A single example is sufficient for the purpose of the discussion. The 'corresponding' equilibrium co-linear configuration is obtained for $d s / d z=2.284083347$. As mentioned before, when starting with two distant, incoming hetons whose vortices are initially aligned in the $y$-direction (translating in the $x$-direction), we should not expect the value of $d s / d z=$ 2.284083347 to correspond to a uniform rotation. This indicates nevertheless that we should test values of the ratio $d s / d z$ around 2 . The numerical experiment indicates indeed that a temporary global rotation is achieved for $d s / d z$ near 2 . This is illustrated in figure 5 . For the value $d s / d y=2.0023$, the point vortices have achieved one full loop, before escaping at an angle as hetons. However, we can see by comparing the trajectories with neighbouring case with $d s / d z=2$ and $d s / d z=2.01$ we see that

- only one loop is achieved for $d s / d z=2.0023$,

- any very small change in $d s / d z$ has a significant effect on the topology of the trajectories.

Arguably, finer tuning of $d s / d z$ may lead to several loops before the vortices escape. But that would still mean that any perturbation, even at infinitesimal level, is likely to modify the trajectories. To further study the formation of Z-vortices, we next study the nonlinear evolution of finite-core vortices. Before focusing on the evolution of finite-core hetons using the full quasi-geostrophic dynamics, we use a model which may be seen as intermediate between the point vortex approach and the full dynamics: the Ellipsoidal 

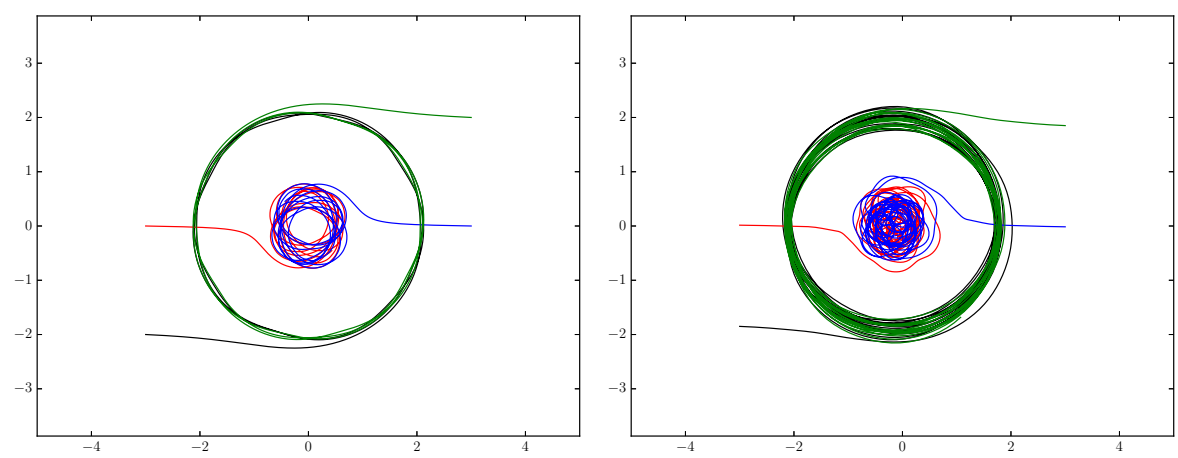

Figure 6 . Top view of the trajectories of interacting, ellipsoidal hetons with $d y / d z=2$ and $d z / h=1$. On the left, the inital vortices are sphere $(r / h=0.5)$. Here, $d s / d z=2$, and $d x / d z=6$ at $t=0$. Right, the initial vortices are spheroids of same height (keeping $d z / h=1$ ) but twice the horizontal radius $(r / h=1)$, while $d s / d y$ is reduced to 1.7 .

Model developed by Dritschel, Reinaud and McKiver (2004). In this model, the vortices are modelled by deformable ellipsoids of uniform PV. This is the first refinement from a singularity located at the centre of vorticity to model a finite-core vortex. The ellipsoids may deform as a consequence of the shear and strain induced by the other vortices. These deformations are the dominant ones and all, non ellipsoidal deformations are filtered out by construction. This approach have proven to be extremely accurate, see Dritschel et al. (2004), and have been used, for example, to determine the critical merger distance between two co-rotating vortices (Reinaud and Dritschel, 2005). One caveat of the model is its inability to model the filamentation and/or the breaking up of vortices. In the Ellipsoidal Model, vortices remain ellipsoids of constant volume at all time.

We reproduce the previous example replacing the singularities at $t=0$ by finite volume spheres of aspect ratio $r / h=r / d z=0.5$. Here, $h$ represents the full height of the vortices and $r$ their horizontal radius. This means that the vortices are adjacent in the vertical (in a layered model, the vortices would be in adjacent layers $i$ and $i+1$ ). Recall that a sphere of PV produces mathematically exactly the same external velocity field as a singularity of same strength located at its centre. The trajectories are presented for $d s / d z=2$ in figure 6 (left). We see a significant difference from the point vortex calculation in which the vortices were escaping as dipoles, see figure 5 (top-right). The vortices have quasi-periodic, quasi-circular trajectories. This motion persists in time (and continues by the time of the end of the calculation). The wobbling of the shape of the nearly spherical vortices stabilises the rotation. This may be due to the fact that the deformation of the vortices induces a small displacement to their respective centroids, correcting their trajectories. The important outcome is that the translation of the hetons (or dipoles) associated with transport over long distances in the oceans is stopped, and the advection remains local, confined within a small area. Because the overall strength of the quartet is zero, the distant environment is quiet. The point vortex calculation provides an indication on the location of the the vortex centres to achieve a meta-stable Z-vortex. vortices of various size and shape but located at the same relative distances of one another may lead to the same behaviour. In practice, this is true within small variations. These variations are the consequence of slightly different dynamical behaviour prior to the hetons encounter. Arguably, in the case of very flat vortices $(r / h \gg 1)$, the hetons may be sensitive to baroclinic instability. The stability of hetons in continuous stratified fluids is addressed in Reinaud (2015). Stable hetons may be obtained for moderate aspect ratios and when the vortices are well separated in the vertical and the horizontal. Recall that 
the point vortex model only provides information of the relative separation distances, since the overall problem can be rescaled in time by $\kappa / d^{3}$, where $\kappa$ is the strength of the singularities and $d$ a separation distance. For large separation for the finite-core vortices, the vortices would resemble singularities. And the point vortex calculation has shown that Z-vortices are extremely difficult to achieve in practice as the tuning of the parameters would become close to the machine precision. Hence, one can deduce that obtaining stable Z-vortices is easier when the ratio of the vortices size to their typical separation distance is not too small. We experiment this by replacing the spherical vortices of the previous case by vortices of the same height by twice the radius $(r / h=1)$. The result is presented in figure 6 (right). In this case we obtained a meta-stable Z-vortex for $d s / d z=1.7$. This is less than in the previous case. The reason for this is a different trajectory during the phase the hetons collide. To understand the trend, it should first be noted that (1) the corresponding collinear state has $d s / d z \sim 2.28$ as seen previously, (2) $d s / d y>\lambda_{c}=0.625$. The latter point means that the hetons are initially deflected such that $d s / d y$ increases. This means that for the hetons to reach the collinear critical separation distance, they need to be located such that $d s / d y<2.28$, which is the case in the two tests presented. In the case where $r / h=1$, the deflection of the vortices is larger. This is due to the fact that the vortices are more spread in the horizontal than in the former case with $r / h=0.5$ and are deflected to avoid colliding into each other. In other words, the hetons induce onto one another a stronger deflection.

It should be noted that this experiment can be repeated over very large sections of the parameter space. Given $d y / d z$, one first can use the two aforementioned criteria to estimate the conditions of formation of the Z-vortex, i.e. a possible range for $d s / d y$. This explicit time-integration of the trajectory of point vortices allows then to estimate the deflection of the hetons prior collision and provides a better estimate for the initial separation $d s / d y$. This value of $d s / d y$ has only a weak dependence on the (arbitrary) choice of the initial horizontal separation $d x$ between the hetons, as far as $d x$ is not too small. Indeed, large $d x$, the hetons (which have overall zero strength) merely interact, and their trajectory is similar to isolated hetons. Only when the hetons are becoming close to one another, does their deflection take place. Recall that in practice this deflection is likely to be an increase in their separation $d s / d y$ in the range of parameters considered. Finally the calculation using the ellipsoidal model allows to obtain meta-stable states. More examples of meta-stable states are available in Appendix III. Each calculation is very rapid, and the limitation resides, in fact, in the storage of the amount of data which can be produced, at very low cost.

It is also interesting to further illustrate the interactions on generic examples solving the full quasi-geostrophic equation. These will allow for deformation of the vortices beyond the ones consistent with ellipsoidal modes. In particular, it allows for destructive interaction during which the vortices may break into smaller vortices and/or shed debris and filaments. These additional dynamical events may be important for (1) generation of small scales (such as sub-mesoscales vortices), and may also affect the overall dynamics. This is the focus of the next section.

\subsection{Full quasi-geostrophic dynamics}

We next focus on the nonlinear evolution of the hetons solving the full quasi-geostrophic dynamics. In all calculations, the potential vorticity is set to $q= \pm 2 \pi$. For reference, it should be noted that a sphere of potential vorticity $q$ has a self-rotation period of $T=$ $6 \pi / q$, while it is $T=4 \pi / q$ for an infinite column. The nonlinear simulations are performed using Contour Dynamics, see Dritschel and Saranavan (1994). The code derives from Contour Surgery by Dritschel (1989) in two dimensions. The domain is discretised by 


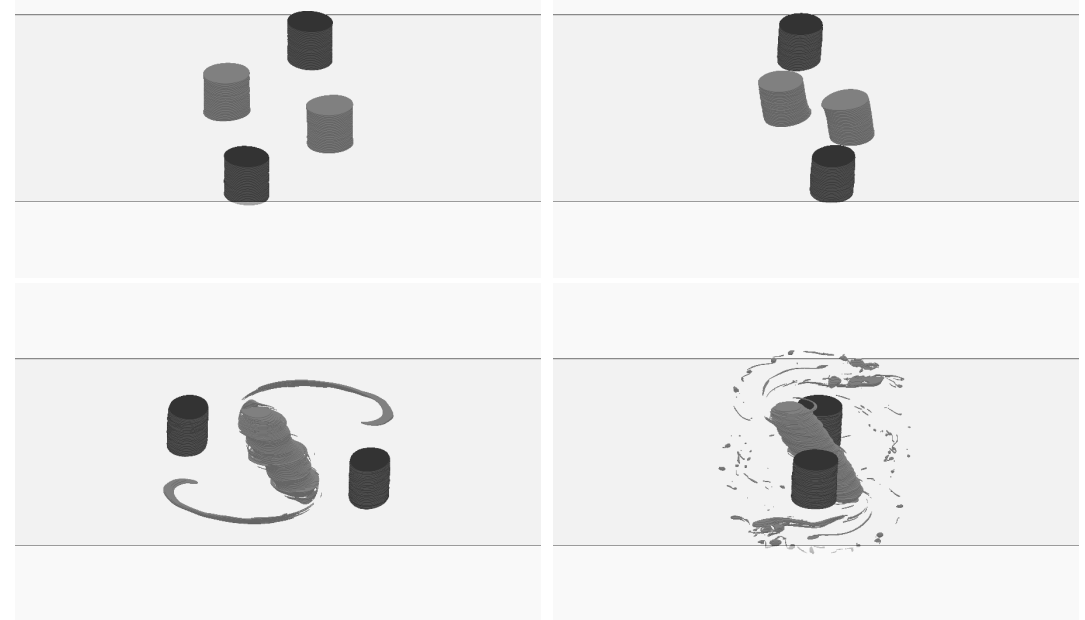

FIgURE 7. PV for the nonlinear evolution for two interacting hetons with $\rho=0.5, \ell z=0, d_{y}=4$ and $d_{s}=1$ such that point equivalent is $d z=1, d y=2$ and $d s=2$. Time displayed (from left to right then top to bottom) are $t=25,37.5,60$ and 92.5 . The hetons are viewed orthographically at an angle of $60^{\circ}$ from the vertical.

100 layers ranging from the bottom of the lower vortices to the top of the upper vortices. Because the method is purely Lagrangian, there is no fixed computational box attached to the problem, and the boundary conditions are imposed at infinity (namely, $\psi$ and $\mathbf{u} \rightarrow 0$ ). This means that the pair of hetons behave as an isolated system without suffering from the interaction of, for example, periodic images. In practice this means that all the resolution effort is on the vortices solely, as the vortices do not need to be confined in small domain to avoid boundary effects. This argument is also valid for the point vortex calculations as well as for the Ellipsoidal Model calculations.

A large number of cases has been investigated, and a summary of the description of the outcome is provided in the Appendix III. We shall limit here the detailed description of the interaction on a selection of generic cases. For consistency, we first illustrate the evolution of the hetons using similar initial conditions as the cases previously studied. Each heton consists of two identical cylindrical vortices of opposite polarity. The vortices have uniform PV. Each vortex has a height $h$ and a radius $r$. Note that the choice of cylindrical vortices instead of ellipsoidal ones is somehow arbitrary. This choice is motivated by previous studies in two-layer models. The two-layer model is constrained to this geometry by construction. Similar results can be obtained using ellipsoidal vortices for the initial conditions instead. We do not expect fundamental dynamical differences from experience (see, for example, Reinaud and Carton, 2009).

We start by simulating the interaction between two hetons with $d y / d z=2$ and $d s / d z=2$. For the first case, we use again $r / h=0.5$ with $d z / h=1$. Using the parametrisation defined in Reinaud and Carton $(2015 \mathrm{a}, \mathrm{b})$, this corresponds to $d_{y}=$ $d y / r=(d y / d z) .(d z / h) .(r / h)^{-1}=4$ and $d_{s} \equiv d s / d y=1$. The parameter $\ell_{z}$ in Reinaud and Carton $(2015 \mathrm{a}, \mathrm{b})$ is the non-dimensional vertical gap between the two vortices, i.e. a measurement of the thickness of the layer of fluid with no potential vorticity between the two vortices, $\ell_{z} \equiv(d z-h) / h$. In this case, since $d z / h=1, \ell_{z}=0$. Results are shown in figure 7. Again, similarly to what is obtained using the Ellipsoidal Model, the vortices form a meta-stable quadrupole which rotates locally. In fact, numerical experi- 


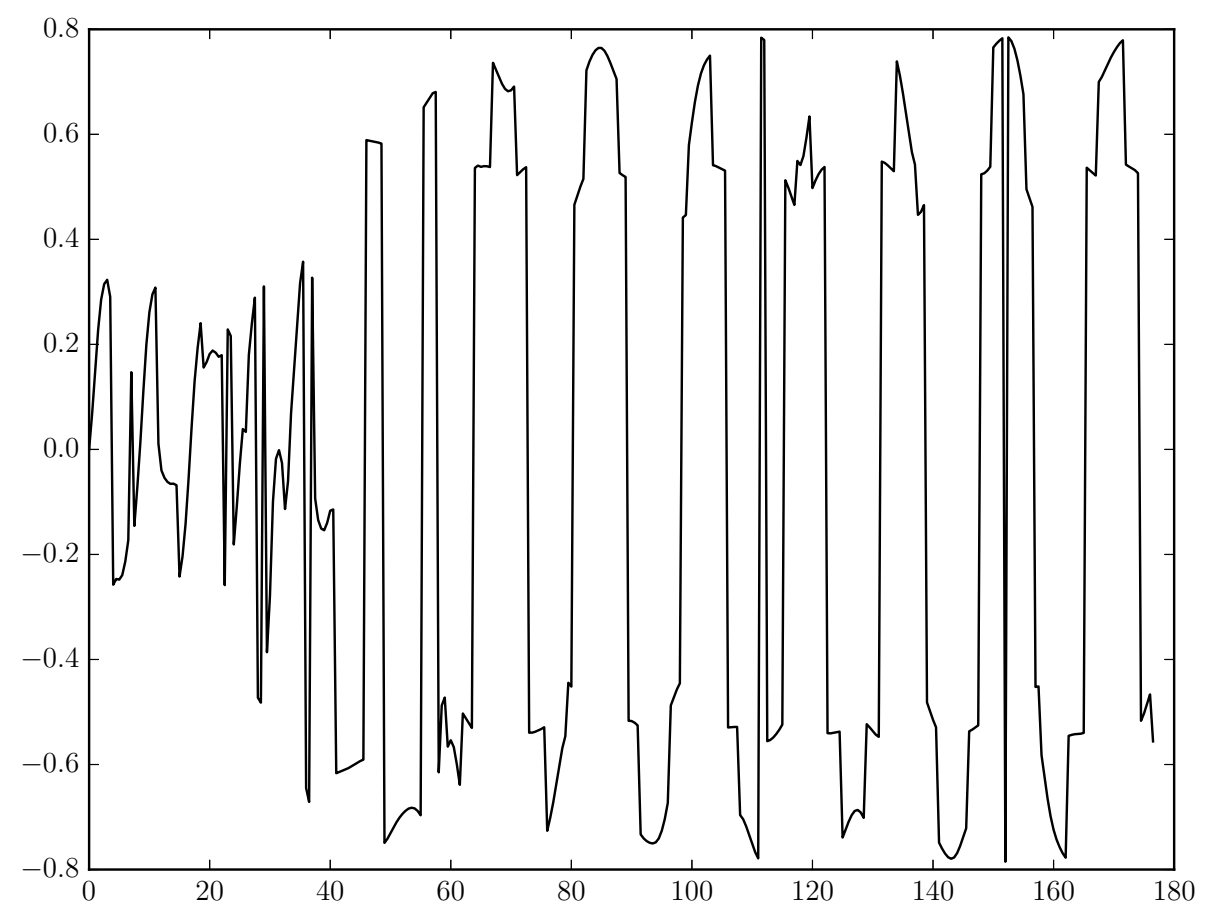

Figure 8 . Time evolution of the angle from the vertical (tilt) $\theta_{\text {tilt }}$ of the largest structure for $\rho=0.5, \ell z=0, d_{y}=4$ and $d_{s}=1$.

ments suggest that quadrupoles are more often obtained when solving the full dynamics. An additional nonlinear effect can be captured when solving the full equations, as nonellipsoidal deformations are then also allowed to occur. Small debris and filaments may be shed from the main structures. This has potentially a strong influence on the development of the flow, as shedding filaments away from the main structures allows for more deformation and motion while preserving some of the flow invariants such as the energy, the linear and angular impulses.

We next illustrate two effects which can be captured within the context of the continuous stratification. Similar results would be obtainable using a many-layer model, but not the classical two-layer model previously used in the literature. We use the fact that we have many degrees of freedom in the vertical direction available to focus on some general aspect of the vertical structure of the flow. The first diagnostic measured is the tilt angle from the vertical of the main structure. Indeed, because of the nature of the Green's function, the advecting velocity field is not uniform with height within the structure, but varies three-dimensionally, despite being constrained to horizontal components. Hence, the bottom of a vortex does not necessarily moves like the top, and the structure may tilt. It is indeed clear from the PV snapshots displayed in figure 7 that the vortices are not rigid, up-standing columns. They tilt and deform in the vertical direction with time. The time evolution of the angle is presented in figure 8. It exhibit oscillation of nearly $\pm 0.8 \mathrm{rad}$, so nearly $\pm 45^{\circ}$.

We next illustrate the formation of debris and filament. The formation of debris and filaments is important as it plays a key role in the energy transfers in physical space, with natural implication on the energy cascade in spectral space. We call debris and filament, any contiguous volume of potential vorticity whose size is less than half the 


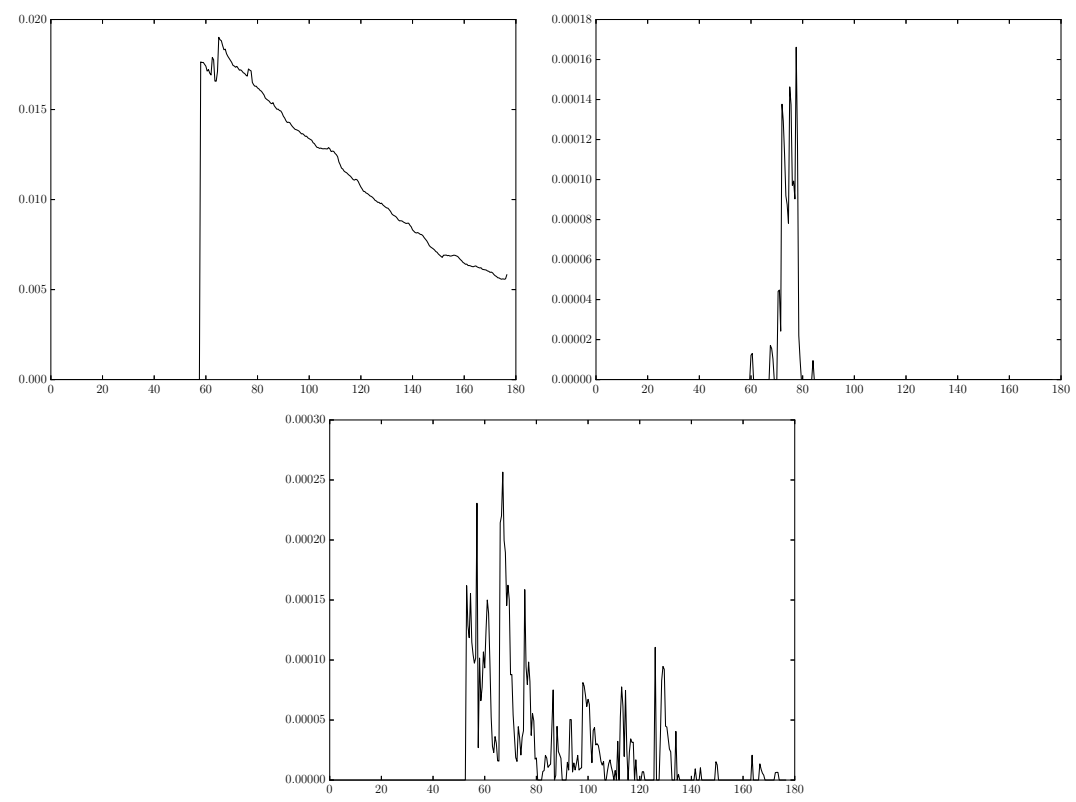

Figure 9. Fraction of the volume $V_{d} / V_{t}$ of debris emitted in the lower third of the bottom vortices (first graph), middle third (second graph), and upper third (last graph) vs time for $\rho=0.5, \ell z=0, d_{y}=4$ and $d_{s}=1$.

initial volume of the original vortices. This choice avoids including the vortices themselves in the analysis, while allowing to include smaller fractions of the vortices such as satellites when they are formed by partial breaking of the vortices. Again, we see from the flow pictures 7, that the shedding of filaments, debris and satellites is not uniform in height. Some horizontal levels are more likely to break and shed debris than others. The picture suggests that most of the debris are in fact shed near the bottom of the quadrupole and or (by symmetry) near the top. To quantify this trend, we decompose the vertical direction in six regions where filaments may be found. Recall that the lack of vertical advection means that filaments can only be found at horizontal levels initially occupied by the vortices. We therefore decompose the vertical direction by splitting the two regions occupied by the lower and upper vortices into three regions each (bottom, middle and top) each corresponding to the first lower third of a vortex, the middle (second third) of the vortex and the upper third of the vortex. The results are shown in figure 9 for the three regions occupied by the lower vortices. By symmetry with respect to the middle horizontal plane $z=0$, the distribution of debris is similar for the upper vortices, with the top part of the upper vortex being similar to the bottom part of the lower vortex. For each instant of time, we calculate the volume of debris $V_{d}$ found in each region. The total volume of the four vortices is denoted $V_{t}$. Although it appears that the first debris identified are near the top of the lower vortices (resp. bottom of the upper vortices), it is confirmed that most of the debris are in fact emitted near the bottom (resp. top) of the flow. The volume decreases with time mostly because they consist in filaments which are continuously stretched by the quadrupole. Eventually the filaments become thinner than the surgery scale and are 'diffused' (removed). At this stage, they are dynamically negligible.

Finally we illustrate a similar case using the same configuration but replacing the vortices by vortices twice as wide with $\rho=1$. Results are presented in figure 10. Here, 


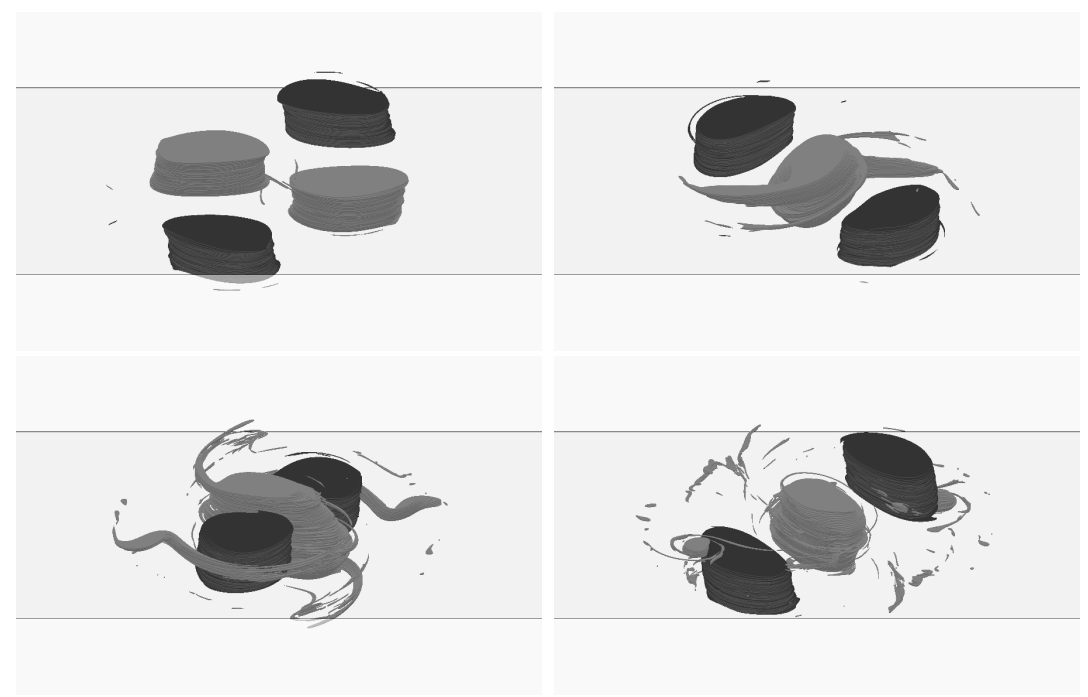

FiguRE 10. PV for the nonlinear evolution for two interacting hetons with $\rho=1, \ell z=0, d_{y}=2$ and $d_{s}=1$ such that point equivalent is $d z=1, d y=2$ and $d s=2$. Time displayed (from left to right then top to bottom) are $t=15,25,35$ and 50 . The hetons are viewed orthographically at an angle of $60^{\circ}$ from the vertical.

we take again $d_{s}=1$. The compound structure is formed again in this case from $t=17$, and persists until the end of the simulation $t=400$, with no indication of breaking apart. Here, and because $\ell_{z}=0$, i.e. there is no vertical gap between to bottom of the upper vortices and the top of the lower vortices, the two inner poles lie directly on the top of one another, and can be seen as a single, contiguous volume of PV. This means that the main structure can be, in fact, seen as a tripole in this case. But this is just a direct consequence of $\ell_{z}=0$.

Finally, we summarise the influence of both the vortices aspect ratio and the vertical gap $\ell_{z}$ between the vortices. The results are summarised in figure 11, where the outcome of the interaction is indicated by a symbol for two values of the aspect ratio $(\rho=0.5,1)$, and three values of the vertical gap $\left(\ell_{z}=0,0.5\right.$, and 1$)$, while keeping the relative centroid separation $d y / d z$ the same (in all cases $d y / d z=2$ ). We then vary $d s / d y$ in the region where the Z-vortex is expected to form (from the results shown above). The most drastic effect of the increasing the vertical gap is to reduce the range where the formation of a Z-vortex is likely. Note that $\ell_{z} \neq 0$ does not prevent completely the formation of $\mathrm{Z}$ vortices. The formation of $\mathrm{Z}$ vortices is still possible (see for example for $\rho=0.5$, $\ell_{z}=1$ and $d s / d y=0.9$, and further examples for wider vortices in Appendix III). The regime becomes however unlikely for small values of $\rho$ (i.e. needs a very fine tuning of the parameter $d s$. in other words only exist for a very small range of $d s$ ). This can be seen as a stronger decoupling in the vertical. One may observe that by increasing the gap $\ell_{z}$ the vortices may resemble more as point vortices (in their interaction in the vertical), and we know that the $Z$ vortex is not exactly reachable from the interaction of incoming hetons in the point vortex theory, but is the result of a unique combination of $d s / d y$ for a given $d z / d y$. On the other hand, increasing the radius of the vortices while keeping the centroid relative position the same enhances the possibility of the formation of the $\mathrm{Z}$ vortex. This is a direct consequence of the fact that PV is not more spread in the horizontal and there is a better horizontal overlap (or horizontal proximity) of the PV at different depths: the coupling between the various vortices is stronger. 

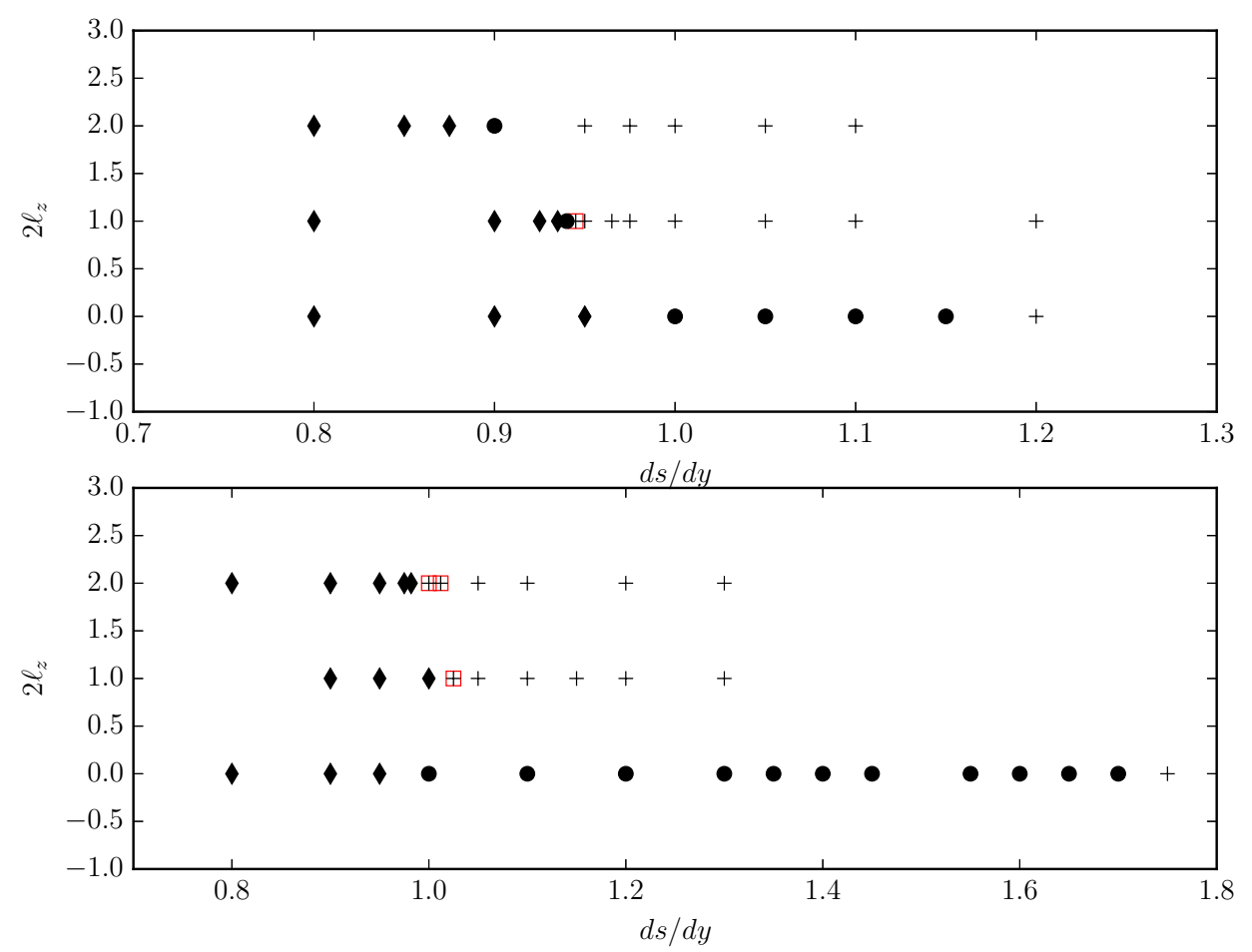

FiguRE 11. Outcome of the nonlinear interactions between the two hetons in the plane $\left(d s / d y, \ell_{z}\right)$. In all cases $d y / d z=2$. The top figure corresponds to $\rho=0.5$, while the bottom figure is for $\rho=1$. indicates a recombination as dipoles, $\bullet$ the formation of a $\mathrm{Z}$ vortex, and + indicates the deviation of the hetons. A red box indicates that a $\mathrm{Z}$ vortex tends to form but breaks rapidly - indicating the possible presence of meta-stable $\mathrm{Z}$ vortex configuration in the vicinity in the parameter space (not reached in practice).

More illustrations of the formation of Z-vortices are presented in Appendix III. However, they follow the same pattern. The $\mathrm{Z}$ vortex is formed when the horizontal offset between the two incoming hetons $d s$ is slightly less that the corresponding critical separation distance for the existence of the collinear equilibrium. This is required since during the initial phase of the approach this distance tends to increase, which can be justified by the criterion 3.1. Eventually, when in the vicinity of one another the vortices may combine to form the quadrupole. This formation and the persistence of the quadrupole are related to both the deformation and the ability to adapt of the vortices, and their finite size which distributed the potential vorticity. Note however, that even spheres, which are nearly equivalent to point vortices, still can form a $\mathrm{Z}$ quadrupole due to their ability to deform.

\section{Finite-core hetons: recombination and deviation}

For the sake of completeness, we propose an illustration of the full dynamics for the two generic regimes where the hetons do not form a quadrupole. In the first one, we illustrate the interaction between hetons which recombine as same depth dipoles. In the second one, the hetons just deviate from their trajectories. Again, these illustrations are generic of all cases in their respective regimes, at least when the initial hetons are stable, and do not break baroclinically before interacting. In the case of breaking hetons, a fraction of 


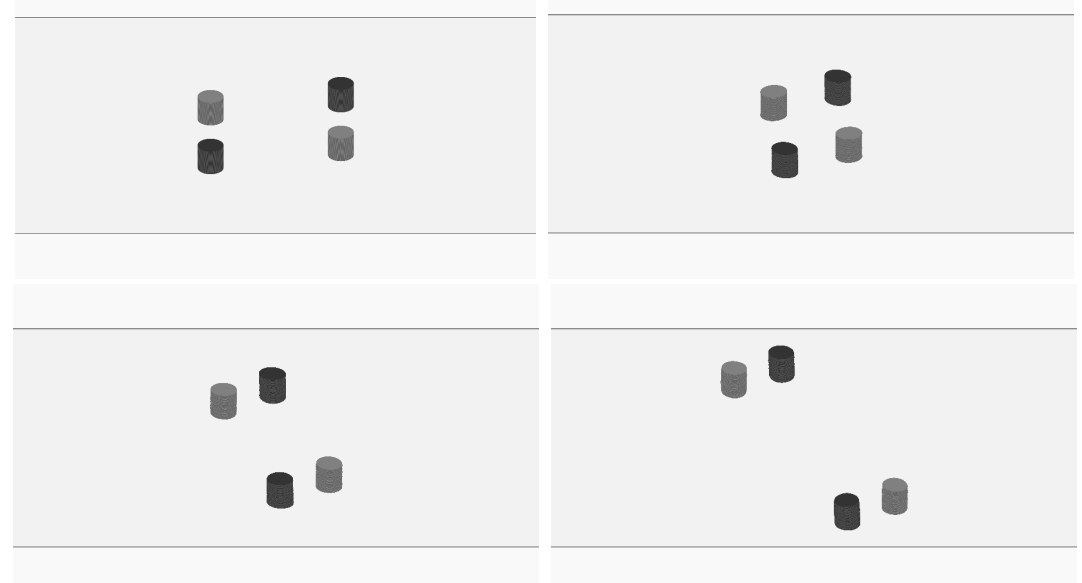

FIGURE 12. PV for the nonlinear evolution for two interacting hetons with $\rho=0.5, \ell z=0, d_{y}=4$ and $d_{s}=0.5$ such that point equivalent is $d z=1, d y=2$ and $d s=2$. Time displayed (from left to right then top to bottom) are $t=0,25,50$ and 75 . The hetons are viewed orthographically at an angle of $60^{\circ}$ from the vertical .

the initial hetons may still interact in a similar way, or the resulting secondary vortices may reconfigure in such a way that there are no longer pairs of hetons moving toward one another. Illustrations of unstable interacting hetons were considered in Reinaud and Carton (2015a) for $d s=0$ and are not further illustrated for small $d s$ in the present paper. For very large $d s$, hetons behave in a similar way as isolated hetons (albeit a small deflection) and their dynamics would be similar to isolated hetons, whose stability properties are known (see Reinaud, 2015).

The first case illustrates the recombination of the vortices as same depth dipoles. Again, we set $d y / d z=2$ and $\ell_{z}=0$, with $\rho=0.5$. This corresponds again to $d_{y}=d y / r=4$. We know that the recombination occurs for small $d s$, hence we take $d s / d y=0.5$. Results are presented in figure 12 . This is qualitatively similar to cases with $d s=0$, apart from the angle at which the dipoles escape. Such cases are detailed in Reinaud and Carton (2015a). Again, it should be noted that these cases are generic of small $d s$ but for cases where the hetons are unstable prior the interaction.

The next case illustrates the final regime where the hetons are horizontally offset not enough to strongly interact but enough to deflect their trajectory as a consequence of the close encounter. This is typically true for offsets large enough compared with the distance between point vortices in the corresponding equilibrium. Results are presented in figure 13 for one case, but they are generic of all such cases for large horizontal offsets.

\section{Conclusion}

This paper has addressed the complex interaction between two hetons which are offset horizontally. We have considered the particular situation when the vortices of the hetons lying at the same depth have opposite polarity, a situation refereed to as 'anti-symmetric' hetons in Reinaud and Carton (2015a). In this context, we have seen that there are, in general, three main regimes of interaction. The kind of interaction crucially depends on the horizontal offset between the axis along which the individual hetons initially translate. The critical values for which we have the transition from one regime to another is a very complex function of all the parameters of the flow, such as the aspect ratio for the 


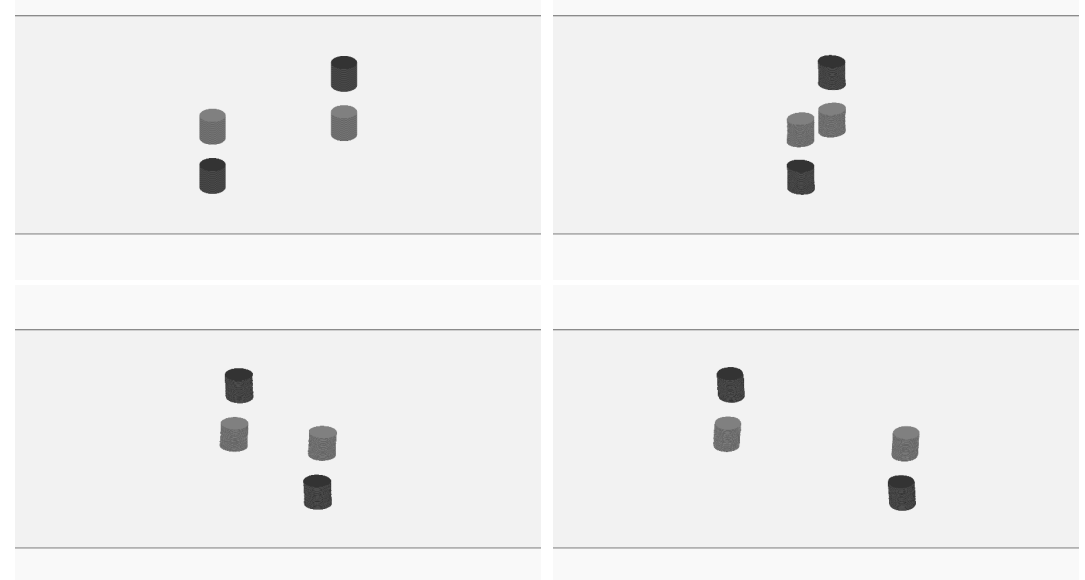

FIGURE 13. PV for the nonlinear evolution for two interacting hetons with $\rho=0.5, \ell z=0, d_{y}=4$ and $d_{s}=2$ such that point equivalent is $d z=1, d y=2$ and $d s=2$. Time displayed (from left to right then top to bottom) are $t=0,25,50$ and 57 . The hetons are viewed orthographically at an angle of $60^{\circ}$ from the vertical.

hetons' vortices $\rho=r h$, the vertical gap between the upper and lower vortices within the hetons $\ell_{z}$, and the horizontal offset between the vortices of the hetons $d_{y}$. There are however, simple ways to estimate a range for the values of the critical offsets separating the different regimes. These ranges can be determined in fact from a simple point vortex calculation. The rationale behind this is as follows. We know that for anti-symmetric hetons, the two incoming baroclinic structures recombine as same-depth dipoles which escape at a right angle when the offset between the two hetons is zero (head-on collision), see for example Reinaud and Carton (2015a). As a consequence, hetons which are only slightly offset will exhibit a similar behaviour and will separate, recombining as dipoles which escape at angle depending on the offset.

For large offsets, the interaction between the hetons becomes weak. Indeed, in all our cases, the net strength of each heton is zero, hence their distant influence is very weak indeed. As a consequence, the hetons will only deviate their trajectory. The deviation becomes smaller as the offset is increased.

These two different behaviours compete in an intermediate range for the horizontal offsets between the incoming hetons. There, the incoming hetons may start to rotate around one another creating a compound quadrupolar structure. Due to its apparent shape, the structure is referred to a Z-vortex. The formation of such a structure is triggered by the existence of an exact equilibrium for a set of four point vortices. In these equilibria, the four point vortices are aligned horizontally (albeit they do not all lie at the same depth) and they rotate uniformly with respect to a vertical axis (which coincides with an axis of horizontal symmetry for the configuration). The exact relative position of the vortices can be obtained, and provides an indication on the conditions of formation of the Z-vortex. For point vortices, the configuration cannot be reached from two incoming singular hetons from afar. This is naturally due to the fact that the point vortices need to lie on the circular trajectories at all time to be in equilibrium (they cannot reach an equilibrium configuration from a non-equilibrium situation without external action). However, it has been shown that finite-volume vortices are capable of forming Z-vortices due to their ability to deform and adapt to the then time-dependent straining field the vortices induce on one another. It has been shown that flatter vortices are more capable 
of adapting, arguably due to the larger horizontal overlap for vortices offset in the vertical. The vertical separation between the vortices of the hetons plays as a deterrent for the formation of the Z-vortex while not preventing it (examples of Z-vortices for offset vortices may be found). The present paper has focused on a limited selection of cases, due to the very large size of the overall parameter space (whose comprehensive investigation is out of reach). The cases presented are in fact generic of the interaction over a large range of parameters. To complement this, the summary outcome of a larger number of cases run over the course of this study is available in the appendix III (section 7.3). Further illustrations of the interactions are also available in Appendix III.

This paper has addressed a complex problem over a wide parameter space, even after having restricting our interest to some idealised situations in which the hetons are similar (all the vortices have same volume and same potential vorticity in absolute value).

There is a natural extension to the problem to more practical issues in the oceans. One may interested at studying the interaction between hetons associated with higher Rossby numbers, which may be relevant to smaller scale structures (submesoscales). This could be done using the closely-related numerical method known as the Non-Hydrostatic Contour Semi-Lagrangian Method, developed by Dritschel and Viudez, see Vìudez and Dritschel (2002) and (2003).

\section{Appendices}

\subsection{Appendix I: Velocities in a regular quadrupole}

This is a crude approach in an attempt to separate the 2 regimes (deflect as hetons or recombine as dipoles). The intermediate regime, when effects compete is when Z-vortices can form. The idea is to focus on the velocity $v$ of one vortex in the inside corner (here vortex 1) and try to see if it will go toward or away from the outside corner, same depth of the second heton (here vortex 4). Of course this does not consider relative displacement at this stage, nor the fact that all distances are time dependent. For sake of simplicity the strength of the point vortices is implicitly scaled by $4 \pi$ to avoid rewriting the factor $1 / 4 \pi$ arising from the Green's function.

Velocity $v$ of point 1

$$
v_{1}=\kappa_{3} \frac{(-d x)}{\left(r_{1,3}\right)^{3}}+\kappa_{4} \frac{(-d x)}{\left(r_{1,4}\right)^{3}},
$$

where $r_{i, j}$ is the distance between vortices $i$ and $j$.

$$
v_{1}=-d x\left(\frac{1}{\left(d x^{2}+(d s-d y)^{2}+d z^{2}\right)^{3 / 2}}-\frac{1}{\left(d x^{2}+d s^{2}\right)^{3 / 2}}\right),
$$

The sign of $v_{1}$ is the one of

$$
-\left(d x^{2}+d s^{2}\right)^{3 / 2}+\left(d x^{2}+(d s-d y)^{2}+d z^{2}\right)^{3 / 2}
$$

which depends only on (to be positive)

$$
d x^{2}+d s^{2}<d x^{2}+(d s-d y)^{2}+d z^{2}
$$

that is

$$
d s^{2}<d s^{2}-2 d s . d y+d y^{2}+d z^{2},
$$




$$
2 d s . d y<d y^{2}+d z^{2}
$$

That is

$$
\frac{d s}{d y}<\frac{1+(d z / d y)^{2}}{2}
$$

For $d s / d y \ll \frac{1+(d z / d y)^{2}}{2}, v_{1}>0$, and one expects an escape as dipoles, as vortex 1 tends to move towards vortex 4 (which will be the second vortex in the pair). Recall that for $d s=0, v_{1}$ is always negative For $d s / d y \gg \frac{1+(d z / d y)^{2}}{2}, v_{1}<0$ the heton is deflected to the right. For $d s / d y \sim \frac{1+(d z / d y)^{2}}{2}$ effects compete in a complex time-dependent way.

Velocity $u$ of point 1

$$
\begin{gathered}
u_{1} \propto-\kappa_{2} \frac{d y}{\left(r_{1,2}\right)^{3}}-\kappa_{3} \frac{d y-d s}{\left(r_{1,3}\right)^{3}}-\kappa_{4} \frac{-d s}{r_{1,4}^{3}} \\
u_{1} \propto \frac{d y}{\left(d y^{2}+d z^{2}\right)^{3 / 2}}-\frac{d y-d s}{\left.\left(d x^{2}+(d y-d s)^{2}+d z^{2}\right)\right)^{3 / 2}}+\frac{-d s}{\left(d x^{2}+d s^{2}\right)^{3 / 2}}, \\
u_{1} \propto d y\left[\frac{1}{\left(d y^{2}+d z^{2}\right)^{3 / 2}}-\frac{1}{\left(d x^{2}+(d y-d s)^{2}+d z^{2}\right)^{3 / 2}}\right]+ \\
d s\left[\frac{1}{\left(d x^{2}+(d s-d y)^{2}+d z^{2}\right)^{3 / 2}}-\frac{1}{\left(d x^{2}+d s^{2}\right)^{3 / 2}}\right] .
\end{gathered}
$$

Limiting cases

- $d s=0$ (known) In that case

$$
u_{1} \propto d y\left[\frac{1}{\left(d y^{2}+d z^{2}\right)^{3 / 2}}-\frac{1}{\left(d x^{2}+(d y-d s)^{2}+d z^{2}\right)^{3 / 2}}\right] .
$$

with is always positive (the hetons go towards each other. The velocity goes to 0 as $\Delta x$ goes to 0 , i.e. as the hetons get closer.

- $d y$ very small

$$
u_{1} \sim d s\left[\frac{1}{\left(d x^{2}+d s^{2}+d z^{2}\right)^{3 / 2}}-\frac{1}{\left(d x^{2}+d s^{2}\right)^{3 / 2}}\right] .
$$

Again, this is 0 when $d x \rightarrow 0$. Otherwise this would be negative and would the dominant term corresponds to the negative (clockwise) rotation of 1 and 4 . This regime is not of interest in this study.

- $d s=d y$

$$
u_{1} \propto d y\left[\frac{1}{\left(d y^{2}+d z^{2}\right)^{3 / 2}}-\frac{1}{\left(d y^{2}+d x^{2}\right)^{3 / 2}}\right]
$$

Initially, for large $d x, u_{1}$ is positive and decreases as $d x$ decreases to $d z$.

\subsection{Appendix II: Z-vortex equilibria for point vortices}

Consider two collinear hetons, see the geometry in figure 14. The four singularities are aligned in the $y-z$ plane. We write down the velocity $u$, perpendicular to this plane for the singularity, assuming that we are placed in a uniform rotation $\Omega$ about the $z$-axis. We are looking for solutions where we can find $u=0$ for all singularities, i.e. the flow is steady. $v=0$ by construction. By symmetry, it is easy to show that $u_{3}=-u_{1}$ and 
$u_{2}=-u_{4}$. Therefore we only need to focus on $u_{1}=0$ and $u_{2}=0$. Each of these equations will give a condition on $\Omega$ which will depend on both parameters $d y / d z$ and $d s / d z$. For the equilibrium to exist, the condition needs to be unique, hence imposing a constraint $f(d y / d z, d s . d z)=0$. The geometry and the parameters are defined in figure 14 .

The equation reads

$$
\begin{gathered}
u_{1}=-\kappa_{2} \frac{\left(y_{1}-y_{2}\right)}{\left(r_{1,2}\right)^{3}}-\kappa_{3} \frac{\left(y_{1}-y_{3}\right)}{\left(r_{1,3}\right)^{3}}-\kappa_{4} \frac{\left(y_{1}-y_{4}\right)}{\left(r_{1,4}\right)^{3}}+\Omega y_{1}, \\
u_{2}=-\kappa_{1} \frac{\left(y_{2}-y_{1}\right)}{\left(r_{1,2}\right)^{3}}-\kappa_{3} \frac{\left(y_{2}-y_{3}\right)}{\left(r_{2,3}\right)^{3}}-\kappa_{4} \frac{\left(y_{2}-y_{4}\right)}{\left(r_{2,4}\right)^{3}}+\Omega y_{2} . \\
u_{1}=-\frac{d y}{\left(d y^{2}+d z^{2}\right)^{3 / 2}}-\frac{(d s-d y)}{\left((d s-d y)^{2}+d z^{2}\right)^{3 / 2}}+\frac{1}{d s^{2}}+\Omega \frac{d s-d y}{2}, \\
u_{2}=-\frac{(d y)}{\left(d y^{2}+d z^{2}\right)^{3 / 2}}-\frac{1}{d s^{2}}+\frac{(d s+d y)}{\left((d s+d y)^{2}+d z^{2}\right)^{3 / 2}}+\Omega \frac{d s+d y}{2}=0 .
\end{gathered}
$$

Adding the two equations and subtracting them gives

$$
\begin{array}{r}
\Omega d s-2 \frac{d y}{\left(d y^{2}+d z^{2}\right)^{3 / 2}}-\frac{(d s-d y)}{\left((d s-d y)^{2}+d z^{2}\right)^{3 / 2}}+\frac{(d s+d y)}{\left((d s+d y)^{2}+d z^{2}\right)^{3 / 2}}=0 \\
\Omega d y+\frac{(d s+d y)}{\left((d s+d y)^{2}+d z^{2}\right)^{3 / 2}}+\frac{(d s-d y)}{\left((d s-d y)^{2}+d z^{2}\right)^{3 / 2}}-\frac{2}{d s^{2}}=0 .
\end{array}
$$

Multiplying the former equation by $d y$ and subtracting the latter multiplied by $d s$ allows to eliminate $\Omega$ and we finally obtain

$$
\begin{array}{r}
\left(\left(\frac{d s}{d z}\right)^{2}-\left(\frac{d y}{d z}\right)^{2}\right)\left(\frac{1}{\left(1+\left(\frac{d y-d s}{d z}\right)^{2}\right)^{3 / 2}}+\frac{1}{\left(1+\left(\frac{d y+d s}{d z}\right)^{2}\right)^{3 / 2}}\right) \\
=2\left(\frac{1}{(d s / d z)}-\frac{(d y / d z)^{2}}{\left(1+(d y / d z)^{2}\right)^{3 / 2}}\right) .
\end{array}
$$

after term cancellations.

Linearising the full equations of motions of the four singularities about the equilibrium (in the reference frame rotating with the equilibrium), we study the linear stability of infinitesimal perturbations $\left(x_{i} \prime, y_{i}^{\prime}\right), i=1,2,3,4$. This provides 8 eigenmodes $\sigma=\sigma_{r}+$ $i \sigma_{r}$ (one for each perturbed variable) where the time-dependence of the perturbation is proportional to $e^{\sigma t}$. The real part $\sigma_{r}$ of $\sigma$ is the growth rate of the perturbation, while its imaginary part is a frequency. Results are shown in figure 15.

It is shown that the is a unstable mode vanishing at $d y \sim 0$, and at $d y \simeq 0.895$. At $d y \simeq 0.895$, we find a classical pattern of exchange of instability where a mode $\sigma=i \sigma_{i}$ with a non-zero frequency which decreases rapidly as $d y$ is decreased. It eventually collapses to zero and the mode becomes unstable. It should be noted that in fact all modes go in pairs. This is if $\sigma$ is eigenvalue, so is $-\sigma$. This means that to all unstable mode $\sigma_{r}>0$, there is a associated stable mode $\sigma_{r}<0$. The negative values of the modes are not represented in the figure. The example for $d y=1$ presented in figure 16 is indeed linearly stable. A example of the trajectories for an unstable case, with $d y=0.7$ is illustrated below. For clarity the trajectory are given in the reference frame rotating at the rotation 


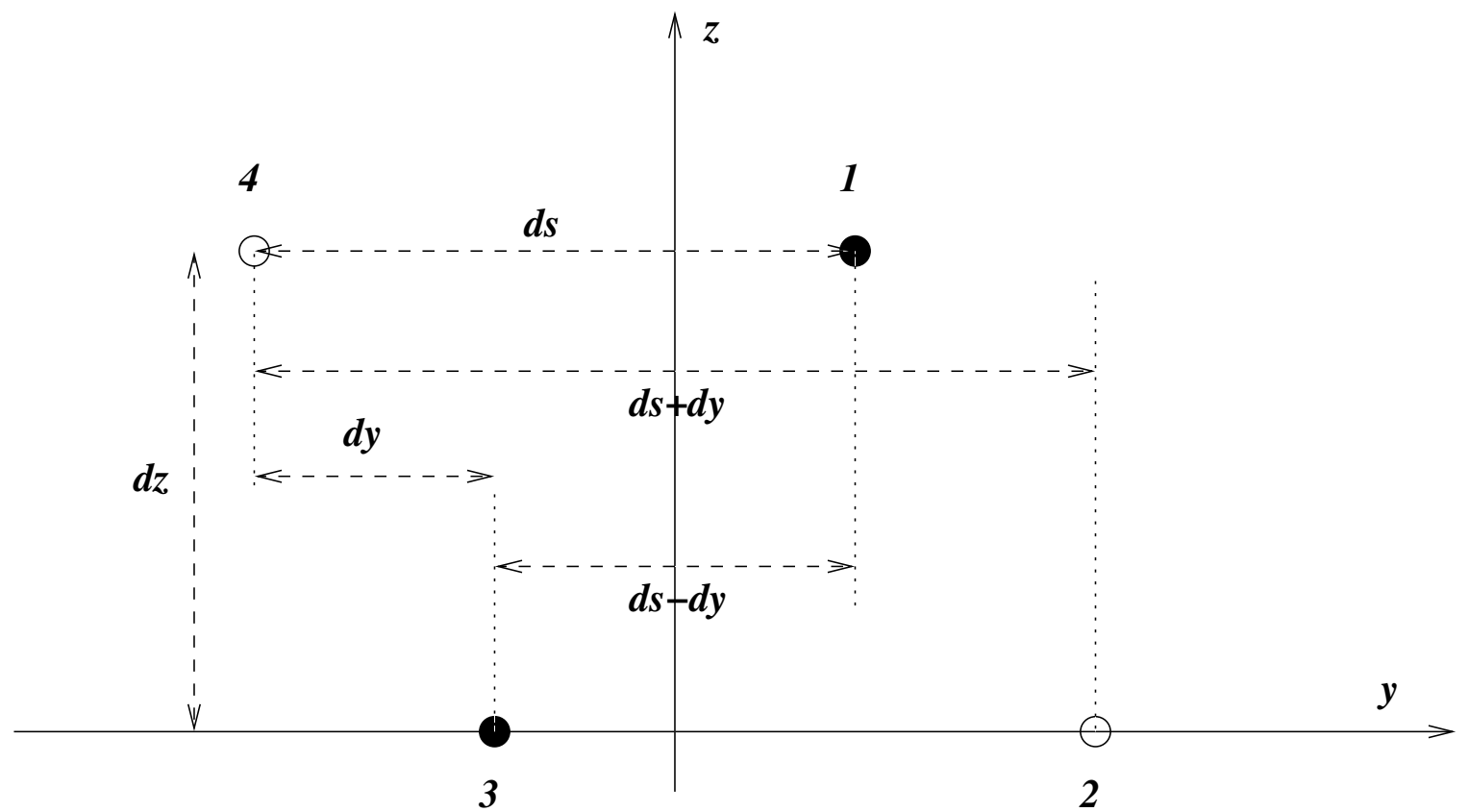

Figure 14. Description of the geometry for the collinear Z vortex. The black disks indicate the two positive vortices.

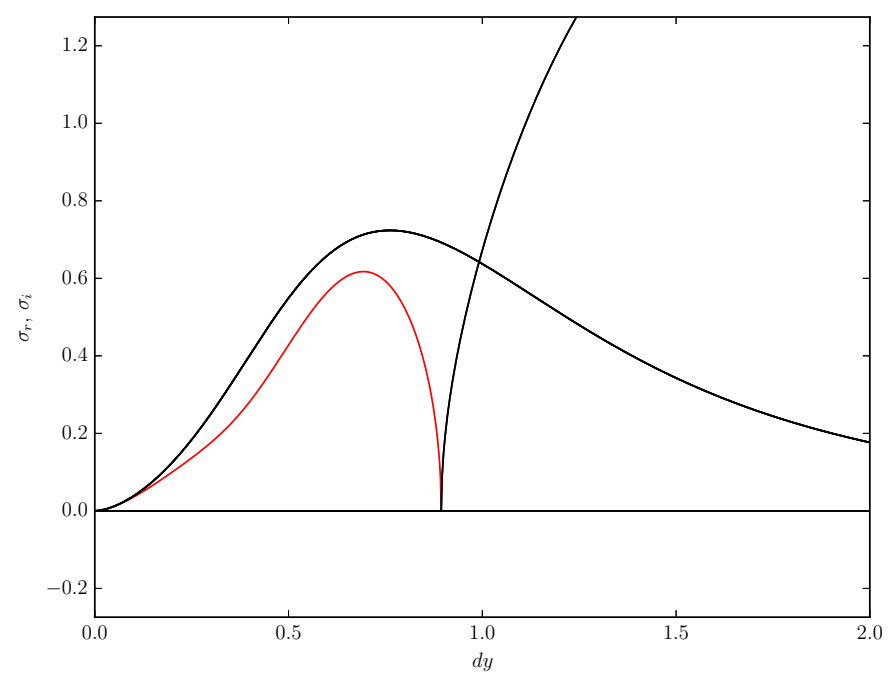

FiguRE 15. Growth rate $\sigma_{r}$ of the most unstable mode (red) vs $d y$ at equilibrium for $d z=1$ (the corresponding value of $d s$ can be obtained from Fig 3), and frequencies $\sigma_{i}$ (black) of neutrally stable modes.

rate of the equilibrium (if the state was stable the trajectories would just be 4 motionless points). 


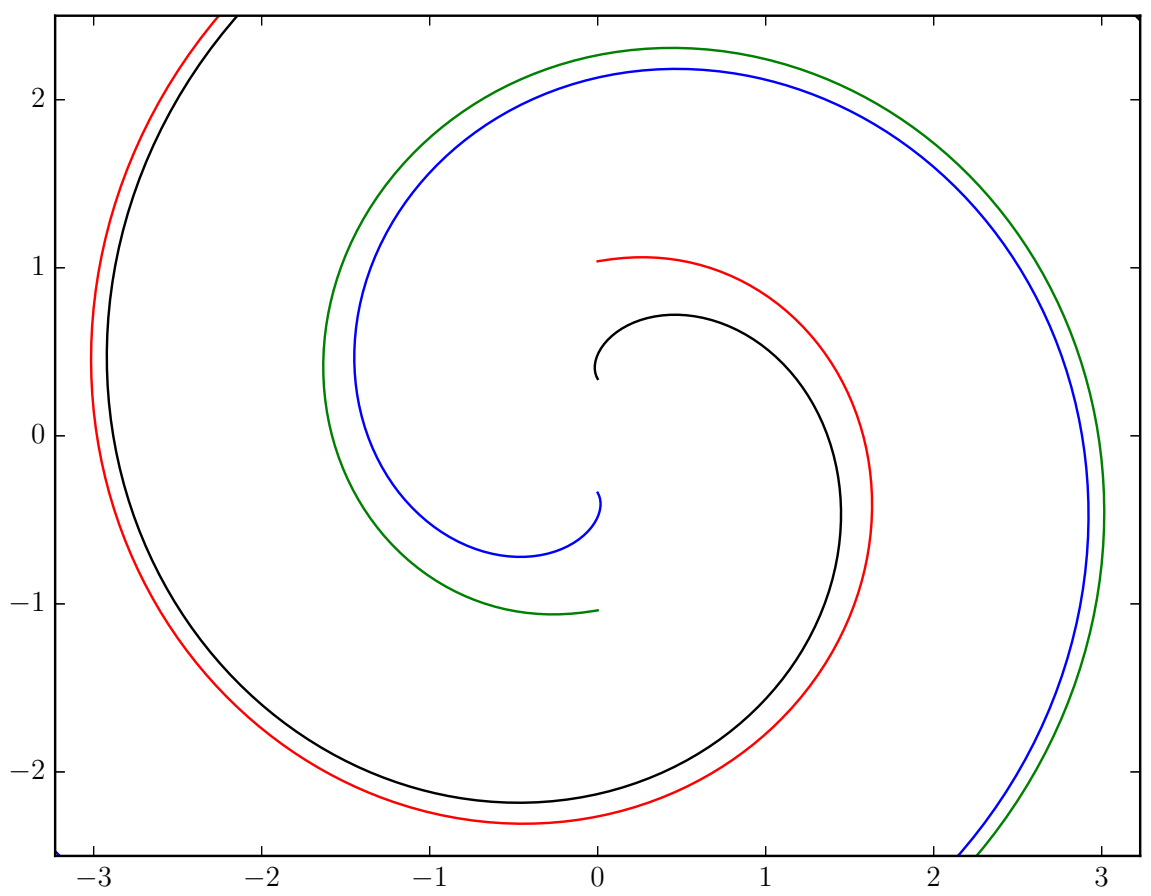

FigurE 16. Trajectories of the unstable point vortex quadrupole equilibria for $d y=0.7$.

\subsection{Appendix III: Outcome of the full nonlinear simulations}

We propose in this appendix tables summarising the outcome of a large series of numerical experiments realised during this research.

we next provide some complementary material to the results presented in the paper.

\subsection{First case, wide vortices: $\varrho=3.5, \ell_{z}=1.5, d_{y}=2$ and $d_{s}=1$}

Table 1 in the appendix III ( $\S 7.3)$ indicates that the case with $\varrho=3.5, \ell_{z}=1.5, d_{y}=2$ and $d_{s}=1$ exhibits the formation of a $Z$-vortex in the full quasi-geostrophic numerical simulation using Contour Surgery. This case has parameters rather different from the ones illustrated in the paper and therefore are worth showning. Indeed, here the vortices are much flatter than in the illustrative case shown in the paper ( $\rho=3.5$ instead of 0.5 or 2). This allowed to find a $Z$ - vortex for a moderate vertical offset. This illustrate further that the formation of a $Z$-vortex, albeit being made more difficult for increasing vertical separation is not prohibited by it.

The set-up means that the vortices centres separations are as follows: $d y / d z=2.8$. The equivalent point vortex collinear equilibria would correspond to $d s / d z=2.82$ (i.e. $d s / d y=0.993 \simeq 1$.

We first illustrate the trajectory of the vortex centres, comparing again the three models available to us, namely point vortex dynamics, the ellipsoidal vortex model and the full quasi-geostrophic dynamics (with Contour Dynamics). Results are presented in figure 17 .

The full quasi-geostrophic evolution is illustrated for this case in figure 18. Its equivalent using the simplified ellipsoidal model is given in figure 19. Both show the formation of the $Z$ quadrupole. 

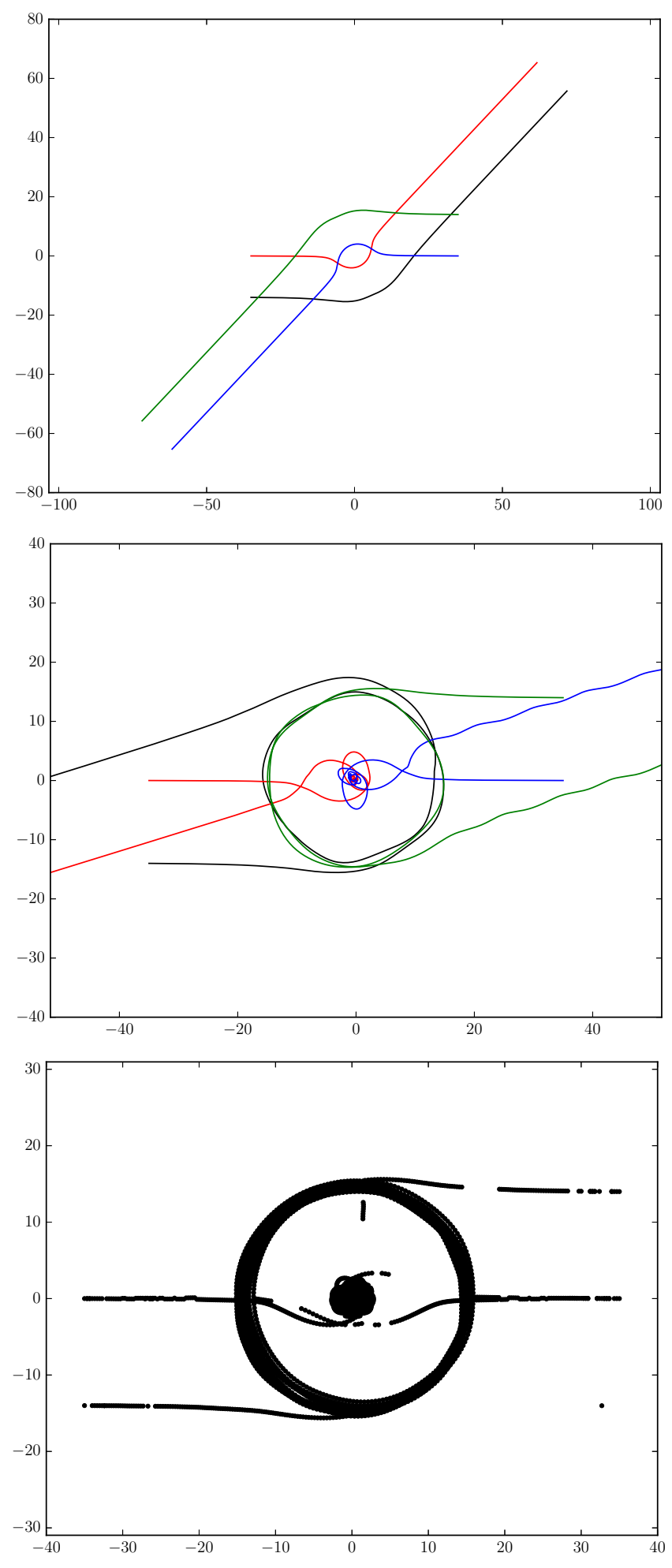

FiguRE 17. Trajectories of the centres of the vortices (four largest vortices in the contour surgery case) for $\varrho=3.5, \ell_{z}=1.5, d_{y}=2$ and $d_{s}=1$. Point vortices (top left), Ellipsoidal model (top right), Contour surgery (bottom). 


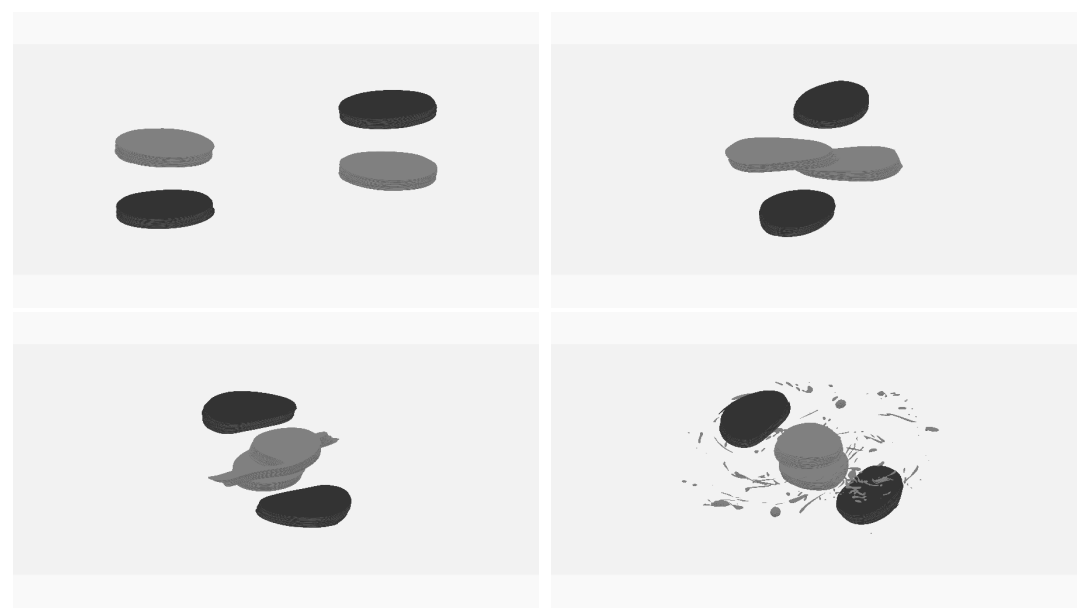

Figure 18. Case $1\left(\varrho=3.5, \ell_{z}=1.5, d_{y}=2\right.$ and $\left.d_{s}=1\right)$ : Snapshots of the evolution of the hetons from the full QG dynamics with Contour Dynamics at time $t=0,50,60,172$.

Again, this shows that the deformation of the vorticess stabilised the quasi-periodic trajectory. In the next figure, we examine in more details the trajectory singular vortices, to illustrate the extreme sensitivity of the singularities trajectories to the set-up.

Going back to the finite-core vortices, and for such a configuration with wide poles, the $Z$-quadrupolar configuration can even been achieved for larger $d_{s}=d s / d y$, as shown below in figure 21 with $d_{s}=2$ (instead of 1$)$.

7.5. Second case: large horizontal offset $d_{y}$, with $\varrho=0.5, \ell z=0, d_{y}=5$ and $d_{s}=1$

In the second case, we go back to the case of hetons with vortices $\varrho=0.5$ illustrated in the main text of the paper (figure 7 of the paper) but with a slightly higher offset between the poles within the hetons. It is shown that the evolution is qualitatively similar, indicating that the case shown in the paper (figure 7 of the paper) is indeed generic. The results are shown in figure 22 .

\subsection{Dipole/Heton}

We next illustrate case where the $Z$-vortex initially forms, but where the strain induced by the vortices onto one another is large enough to destabilise two of the vortices. These vortices break into two pieces which recombine to produce two structures with an $L$ shape. Each structure consists of 3 vortices, and is the combination between a heton (two vortices of opposite sign at different depth, and a dipoles, two vortices of opposite sign at the same depth). This may happen when the vortices are wide enough to break baroclinically. Such a situation is illustrated in figure 23. This example shows that the flow evolution also depends on the stability properties of the structures that form as a result of the interactions. The fate of interacting, wide, hetons in the case $d_{s}=0$ (headon collision), is detailed in Reinaud \& Carton (2015a), see references. This indicates that more complex dynamics may be observed if the structure themselves are sensitive to shear or strain induced instabilities. The systematic and detailed investigation of these behaviours (done for $d_{s}=0$ in Reinaud \& Carton (2015)) is beyond the scope of the present contribution due size of the parameter space with the added degree of freedom $d_{s}$.

\section{REFERENCES}




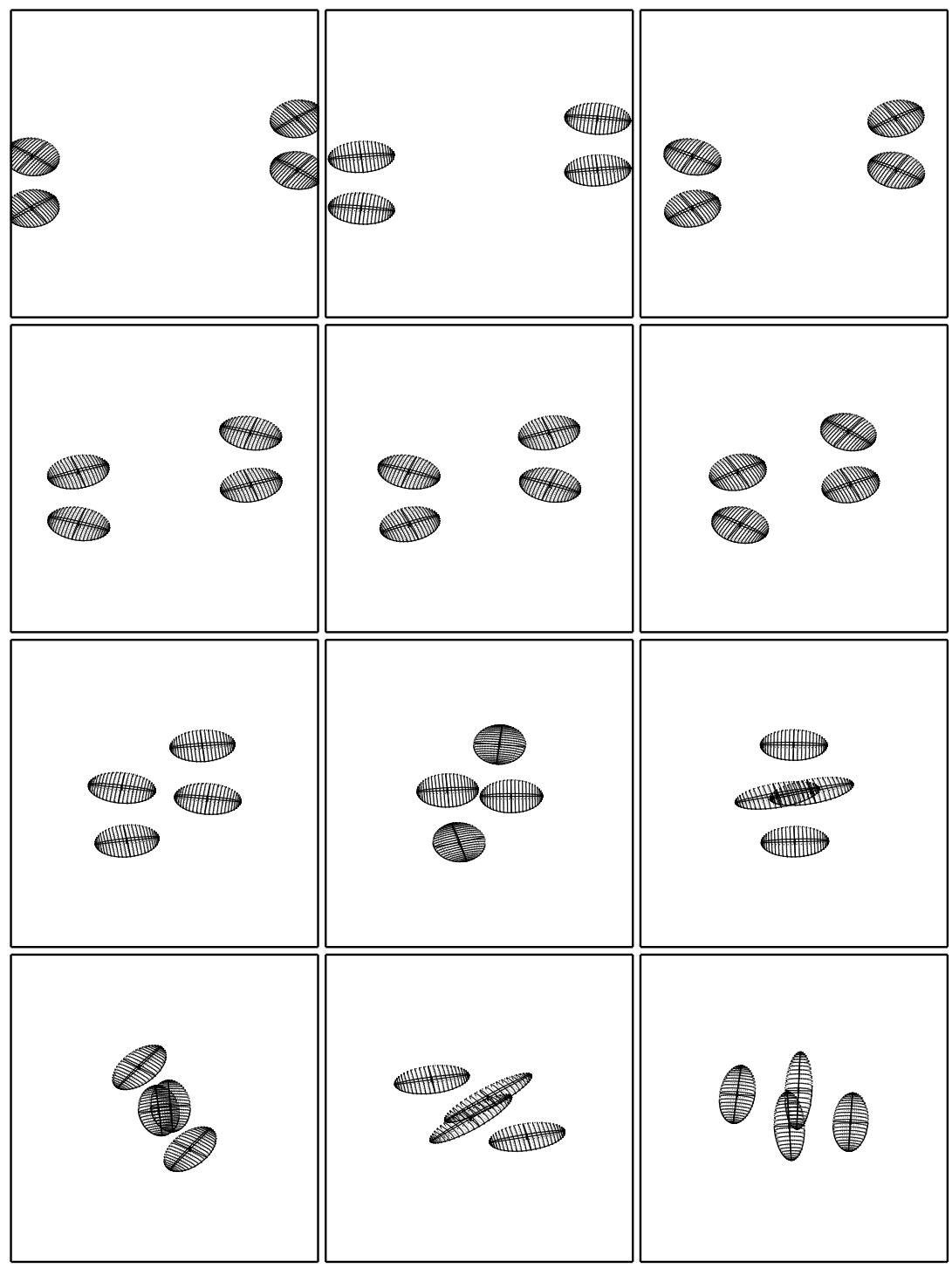

Figure 19. Case $1\left(\varrho=3.5, \ell_{z}=1.5, d_{y}=2\right.$ and $\left.d_{s}=1\right)$ : ELM equivalent.

Carton, X. 2001 Hydrodynamical modeling of oceanic vortices. Surveys in Geophysics 22 (3), $179-263$.

Carton, X., Flierl, G. R., Perrot, X., Meunier, T. \& Sokolovskiy, M. $2010 a$ Explosive instability of geostrophic vortices. part 1: baroclinic instability. Theor. and Comp. Fluid Dyn. 24, 125-130.

Carton, X., Meunier, T., Flierl, G. R., Prrot, X. \& Sokolovskiy, M. $2010 b$ Explosive instability of geostrophic vortices. part 2: parametric instability. Theor. and Comp. Fluid Dyn. 24, 131-135.

Chaо, S.-Y. \& Shaw, P.-T. 1999 Close interactions between two pairs of heton-like vortices under sea ice. J. Geophys Res 104, 23591-23601.

Charney, J. 1947 The dynamics of long waves in a baroclinic westerly current. Meteor. 5 (5), $135-162$.

Chelton, D. B., Schlax, M. G. \& Samelson, R. M. 2011 Global observations of nonlinear mesocale eddies. Prog. Oceanogr. 91, 167-216. 

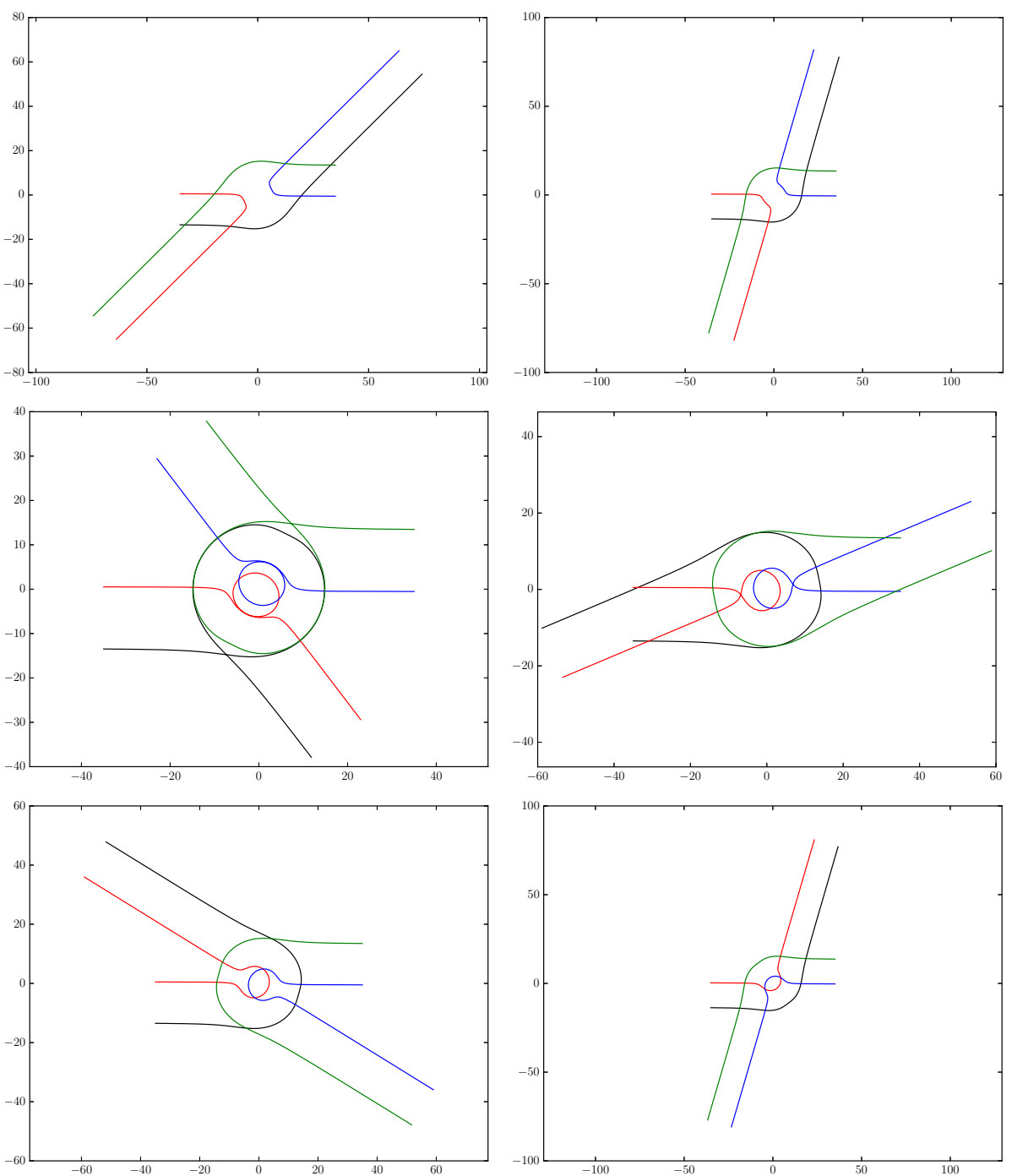

Figure 20. Case I: Point vortex: Examination of trajectories near the formation of the Z-vortex case $1, d y / d z=2.8$, and from left to right then top to bottom, $d s / d y=12.9 / 14,12.94 / 14,12.95 / 14,12.96 / 14,13 / 14, \& 13.4 / 14$.

Chelton, D. B., Schlax, M. G., Samelson, R. M. \& De Szoeke, R. A. 2007 Global observations of large oceanic eddies. Geophys. Res. Lett. 34, L15606.

Chérubin, L., Serpette, A., Carton, X., Paillet, J., Connan, O., Rousselet, B., Le Cann, P., Le Corre, T. \& Labasque, D. 1997 Descriptive analysis of the hydrology and currents on the iberian shelf from gibraltar to cape finisterre : preliminary results from the semane and interafos experiments. Ann. Hydrogr. 21, 5-21.

Dritschel, D. \& Saranavan, R. 1994 Three-dimensional quasi-geostrophic contour dynamics, with an application to stratospheric vortex dynamics. Quart. J. Roy. Meteorol. Soc. 120, 1267-1297.

Dritschel, D. G. 1989 Contour dynamics and contour surgery: numerical algorithms for extended, high-resolution modelling of vortex dynamics in two-dimensional, inviscid, incompressible flows. Computer Phys. Rev. 10, 77-146.

Dritschel, D. G., Reinaud, J. N. \& McKiver, W. J. 2004 The quasi-geostrophic ellipsoidal model. J. Fluid Mech. 505, 201-223. 


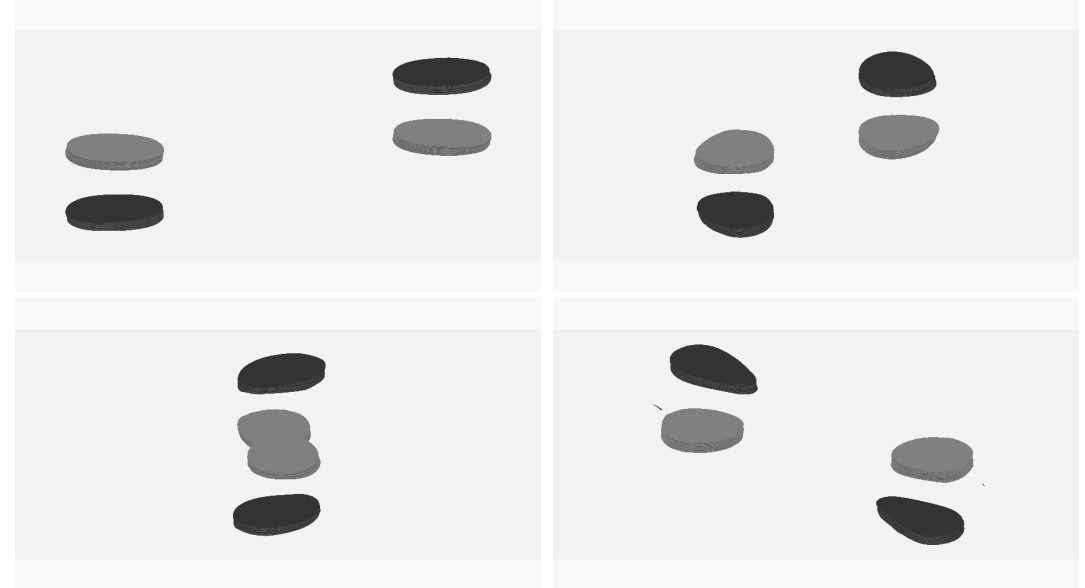

FiguRE 21. Case similar to the case 1 but for a larger horizontal offset $d_{s}$. Here $\varrho=3.5, \ell_{z}=1.5, d_{y}=2$ and $d_{s}=2$. Snapshots displayed at $t=7,32,52,63$.

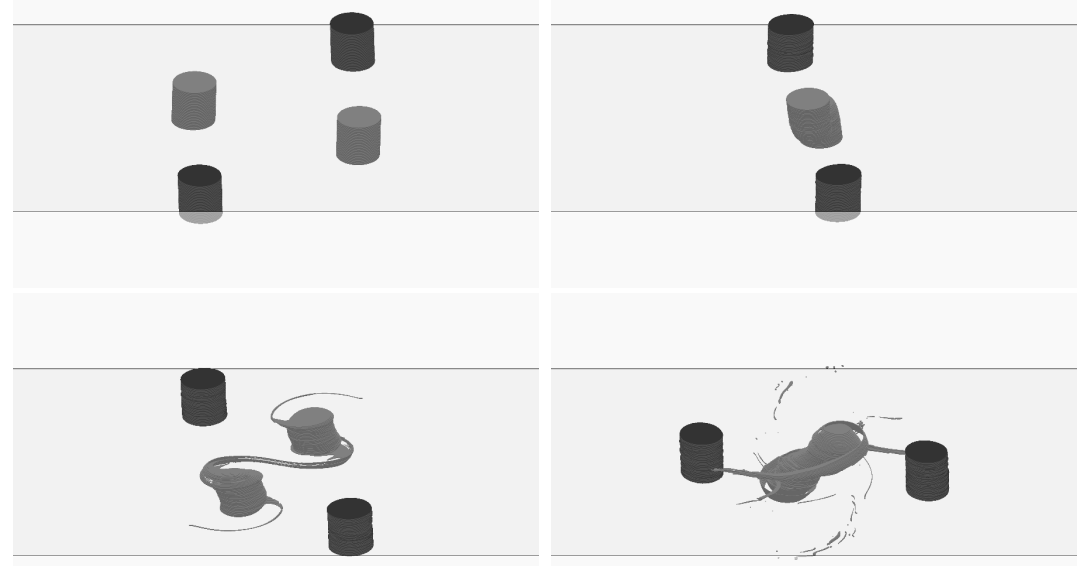

Figure 22. Case 2: CS with $\varrho=0.5, \ell z=0, d_{y}=5$ and $d_{s}=1$.

Dritschel, D. G. \& Vìudez, A. 2003 A balanced approach to modelling rotating stablystratified geophysical flows. J. Fluid Mech. 488, 123-250.

Ebbesmeyer, C. C., Taft, B. A., McWilliams, J. C., Shen, C. Y., Riser, S. C., Rossby, H. T., Biscaye, P. E. \& Östlund, H. G. 1986 Detection, structure and origin of extreme anomalies in a western atlantic oceanogra phic section. J. Phys. Ocean. 16, 591-612.

Flierl, G., Carton, X. \& Messager, C. 1999 Vortex fomation by unstable oceanic jets. Eur. Ser. Appl. Ind. Math $\mathbf{7}$.

FlierL, G. R. 1988 On the instability of geostrophic vortices. J. Fluid Mech. 197, 339-388.

Gryanik, V. M., Sokolovskiy, M. A. \& Verron, J. 2006 Dynamics of heton-like vortices. Regular and Chaotic Dynamics 11, 383-434.

von Hardenberg, J., McWilliams, J. C., Provenzale, A., Shchpetkin, A. \& Weiss, J. B. 2000 Vortex merging in quasi-geostrohic flows. J. Fluid Mech. 412, 331-353.

Helfrich, K. \& Send, U. 1988 Finite-amplitude evolution of two-layer geostrophic vortices. J. Fluid Mech. 197, 331-348.

HogG, N. G. \& Stommel, H. M. 1985 The heton, an elementary interaction between discrete 

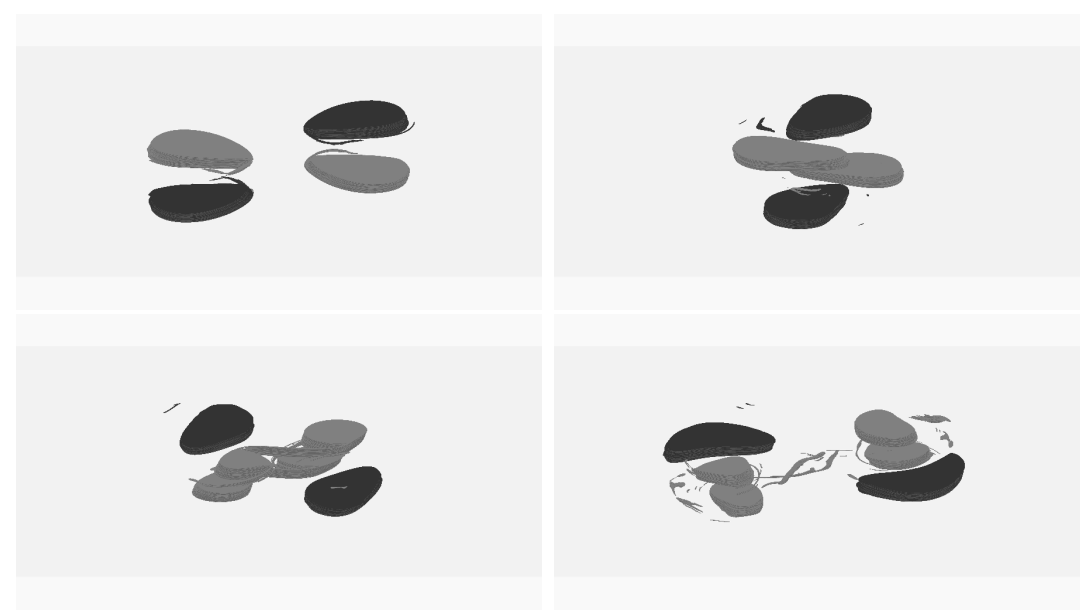

FiguRE 23. Dipole/heton: $\varrho=0.5, \ell z=1.5, d_{y}=1.5$ and $d_{s}=1$. Times displayed are $t=25,40,55,65$.

baroclinic geostrophic vortices, and its implications concerning eddy heat flow. Proc. $R$. Soc. Lond. A 397, 1-20.

KIZNER, Z. 1984 Rossby solitons with axially symmetric baroclinic modes. Trans. of the USSR Acad. of Sci. 275, 211-214.

KIZNER, Z. 2006 Stability and transitions of hetonic quartets and baroclinic modons. Phys. Fluids 18, 056601.

Kizner, Z., Berson, D. \& Khvoles, R. 2002 Baroclinic modon equilibria on the beta-plane: stability and transitions. J. Fluid Mech. 468, 239-270.

Kizner, Z., Berson, D. \& Khvoles, R. 2003 Non-circular baroclinic beta-plane modons: constructing stationay solutions. J. Fluid Mech. 489, 199-228.

Kozlov, V. F., Makarov, V. G. \& Sokolovskiy, M. A. 1986 Numerical model of the baroclinic instability of axially symmetric eddies in two-layer ocean. Izvestiya, Atmos. Ocean. Phys. 22.

L'Hegaret, P., Carton, X., Ambar, i., Menesguen, C., Hua, B., Chérubin, L., Aguiar, A., Le Cann, B., Daniault, N. \& Serra, N. 2014 Evidence of mediterranean water dipole collision in the gulf of cadiz. J. Geophys. Res. 119 (8), 5337-5359.

Reinaud, J. N. 2015 On the stability of continuously stratified quasi-geostrophic hetons. Fluid Dyn. Res. 47, 035510.

Reinaud, J. N. \& CARTon, X. 2009 The stability and non-linear evolution of quasi-geostrophic hetons. J. Fluid Mech. 636, 109-135.

Reinaud, J. N. \& Carton, X. 2015a Head on collision between two continuously stratified quasi-geostrophic hetons. J. Fluid Mech. 779, 144-180.

Reinaud, J. N. \& CARTon, X. 2015b Existence, stability and formation of baroclinic tripoles in quasi-geostrophic flows. J. Fluid Mech. 785, 1-30.

Reinaud, J. N. \& Dritschel, D. G. 2002 The merger of vertically offset quasi-geostrophic vortices. J. Fluid Mech. 469, 287-315.

Reinaud, J. N. \& Dritschel, D. G. 2005 The critical merger distance between two co-rotating quasi-geostrophic vortices. J. Fluid Mech. 522, 357-381.

Reinaud, J. N. \& Dritschel, D. G. 2009 Destructive interactions between two counterrotating quasi-geostrophic vortices. J. Fluid Mech. 639.

Sokolovskiy, M. A. \& Carton, X. 2010 Baroclinic multipole formation from heton interaction. Fluid Dyn. Res. 42, 045501.

Sokolovskiy, M. A. \& Verron, J. 2000 Finite-core hetons: stability and interactions. J. Fluid Mech. 423, 127-154.

SokolovskiY, M. A. \& VerRon, J. 2014 Dynamics of vortex structures in a stratified rotating fluid. Atmospheric and Oceanographic Sciences Library 47, Springer. 
VAlcke, S. \& Verron, J. 1993 On interactions between two finite-core hetons. Phys. Fluids, $A$ 5, 2058-2060.

VAlLis, C. 2006 Atmospheric and Oceanic Fluid Dynamics: Fundamentals and Large-scale Circulation. Cambridge University Press.

Vìudez, A. \& Dritschel, D. G. 2002 An explicit potential vorticity conserving approach to modelling nonlinear internal gravity waves. J. Fluid Mech. 458, 75-101.

Young, W. R. 1985 Some interactions between a small number of of baroclinic, geostrophic vortices. Geophys. and Astrophys. Fluid Dyn. 33, 35-61.

Zhang, Z., WAng, W. \& Qiu, B. 2014 Oceanic mass transport by mesoscale eddies. Science $\mathbf{3 4 5}, 322-324$. 


$\begin{array}{cccc}\varrho & \ell_{z} & d_{y} & d \\ 3 & 1 & 1.5 & 1 \\ & & & 1.5 \\ & 2 & 1.5 & 1 \\ & 3 & 1.5 & 1 \\ & & 2 & 0.5 \\ & 5 & 1.5 & 1.5\end{array}$

Escape as dipoles

Escape as hetons.

Escape as dipoles

Escape as dipoles

Escape as dipoles

Escape as dipoles

$\begin{array}{lllll}5 & 1 & 2 & 0.75 & \text { Positive vortices break into two parts. They move }\end{array}$

$\begin{array}{cccc}3 & 1.5 & 1 & \text { with negative vortex as combined } \\ & & 1.5 & \text { Escape as dipoles } \\ & 2 & 0.5 & \text { Escape as hetons } \\ & 2 & 0.75 & \text { Escape as dipoles } \\ 5 & 1.5 & 1 & \text { Escape as dipoles } \\ & & 1.5 & \text { Escape as dipoles } \\ 7 & 1.5 & 1 & \text { Escape as dipoles } \\ & & 1.5 & \text { Escape as dipoles } \\ & & & \text { Escape as dipoles }\end{array}$

$\begin{array}{lllll}7 & 3 & 1 & 0.5 & \text { Escape as dipoles (positive vortices partially sheared) }\end{array}$

1.51 Positive vortices break into two parts. They move

with negative vortex as combined dipoles/hetons

$0.5 \quad$ Escape as dipoles

$0.75 \quad$ Escape as dipoles

1

$2 \quad 1.125$

$\begin{array}{lll}5 & 1.5 & 1\end{array}$

$1.5 \quad 1.5$

$2 \quad 0.5$

Escape as dipoles

Z-vortex

Escape as dipoles

Escape as hetons

Escape as dipoles

$\begin{array}{lllr}7 & 1.5 & 1 & \text { Escape as dipoles after temporary } \\ \text { Escape as dipoles }\end{array}$

Escape as dipoles

Escape as dipoles

Escape as dipoles

Escape as dipoles

$\begin{array}{lllll}9 & 1 & 1.5 & 1.5 & \text { Baroclinic instability. Partial alignment }(Z-\text { vortex })\end{array}$

$\begin{array}{llll}3 & 2 & 0.5 & \text { Escape as dipoles }\end{array}$

$\begin{array}{llll}3 & 2 & 0.75 & \text { Z-vortex worth two positive satellites }\end{array}$

$\begin{array}{llll}5 & 2 & 0.5 & \text { Escape as dipoles }\end{array}$

$\begin{array}{lll}2 & 0.75 \quad \text { Escape as hetons }\end{array}$

Z-vortex

$\begin{array}{lll}7 & 1.5 & 1\end{array}$

Escape as dipoles

Escape as hetons

Escape as dipoles

Escape as dipoles

Escape as dipoles

Escape as dipoles

Escape as dipoles

Escape as dipoles

TABlE 1. Table summarising the behaviour of a pair of interacting, antisymmetric hetons. 


$\begin{array}{ccccc}\varrho & \ell_{z} & d_{y} & d & \\ 11 & 3 & 2 & 0.5 & \text { Escape as dipoles } \\ & & & 0.75 & \text { Z-vortex with satellites } \\ & 5 & 2 & 0.5 & \text { Escape as dipoles } \\ & & & 0.75 & \text { Escape as dipoles } \\ & 7 & 2 & 0.5 & \text { Escape as dipoles } \\ & & & 0.75 & \text { Escape as dipoles } \\ & 8 & 1 & 1 & \text { Escape as dipoles } \\ & & 1.5 & \text { Partial alignment at centre, dipoles escape. } \\ & & 1.5 & 1 & \text { Escape as dipoles } \\ & 2 & 0.75 & \text { Escape as dipoles } \\ & 1.5 & 1.5 & \text { Escape as hetons } \\ & 11 & 1 & 0.5 & \text { Escape as dipoles } \\ & 1.5 & 1 & \text { Escape as dipoles } \\ & & 1.5 & \text { Escape as dipoles }\end{array}$

TABLE 2. Table summarising the behaviour of a pair of interacting, antisymmetric hetons continued. 